Submitted: May 29, 2018

\title{
AKARI Infrared Observations of the Supernova Remnant G292.0+1.8: Unveiling Circumstellar Medium and Supernova Ejecta
}

\author{
Ho-Gyu Lee ${ }^{1,2}$, Bon-Chul Koo ${ }^{2}$, Dae-Sik Moon ${ }^{3}$, Itsuki Sakon ${ }^{1}$, Takashi Onaka ${ }^{1}$, \\ Woong-Seob Jeong ${ }^{4}$, Hidehiro Kaneda ${ }^{5}$, Takaya Nozawa ${ }^{6}$, and Takashi Kozasa ${ }^{7}$
}

\begin{abstract}
We present the results of $A K A R I$ observations of the O-rich supernova remnant G292.0+1.8 using six IRC and four FIS bands covering $2.7-26.5 \mu \mathrm{m}$ and 50-180 $\mu \mathrm{m}$, respectively. The $A K A R I$ images show two prominent structures; a bright equatorial ring structure along the east-west direction and an outer elliptical shell structure surrounding the remnant. The equatorial ring structure is clumpy and incomplete with its western end opened. The outer shell is almost complete and slightly squeezed along the north-south direction. The central position of the outer shell is $\sim 1^{\prime}$ northwest from the embedded pulsar and coincides with the center of the equatorial ring structure. In the northen and southwestern regions, there is also faint emission with a sharp boundary beyond the bright
\end{abstract}

\footnotetext{
${ }^{1}$ Department of Astronomy, Graduate School of Science, The University of Tokyo, Bunkyo-ku, Tokyo 113-0033, Japan; hglee@astron.s.u-tokyo.ac.jp isakon@astron.s.u-tokyo.ac.jp, onaka@astron.s.u-tokyo.ac.jp

${ }^{2}$ Department of Physics and Astronomy, Seoul National University, Seoul, 151-742, Korea; koo@astrohi.snu.ac.kr

${ }^{3}$ Department of Astronomy and Astrophysics, University of Toronto, Toronto, ON M5S 3H4, Canada; moon@astro.utoronto.ca

${ }^{4}$ Korea Astronomy and Space Science Institute, 61-1, Whaam-dong, Yuseong-gu, Deajeon 305-348, Korea; jeongws@kasi.re.kr

${ }^{5}$ Graduate School of Science, Nagoya University, Chikusa-ku, Nagoya 464-8602, Japan; kaneda@u.phys.nagoya-u.ac.jp

${ }^{6}$ Institute for the Physics and Mathematics of the Universe, University of Tokyo, Kashiwa, Chiba 2778568, Japan; tnozawa@mail.sci.hokudai.ac.jp

${ }^{7}$ Department of Cosmosciences, Graduate School of Science, Hokkaido University, Sapporo 060-0810, Japan; kozasa@mail.sci.hokudai.ac.jp
} 
shell structure. The equatorial ring and the elliptical shell structures were partly visible in optical and/or X-rays, but they are much more clearly revealed in our $A K A R I$ images. There is no evident difference in infrared colors of the two prominent structures, which is consistent with the previous proposition that both structures are of circumstellar origin. However, we have detected faint infrared emission of a considerably high 15 to $24 \mu \mathrm{m}$ ratio associated with the supernova ejecta in the southeastern and northwestern areas. Our IRC spectra show that the high ratio is at least partly due to the emission lines from Ne ions in the supernova ejecta material. In addition we detect a narrow, elongated feature outside the SNR shell. We derive the physical parameters of the infrared-emitting dust grains in the shocked circumstellar medium and compare the result with model calculations of dust destruction by a SN shock. The $A K A R I$ results suggest that the progenitor was at the center of the infrared circumstellar shell in red supergiant stage and that the observed asymmetry in the SN ejecta could be a result of either a dense circumstellar medium in the equatorial plane and/or an asymmetric explosion.

Subject headings: ISM: individual(G292.0+1.8) — infrared: ISM — ISM: dust — shock waves — supernova remnants

\section{Introduction}

Young core-collapse supernova remnants (SNRs) in the Galaxy provide an unique opportunity to study fine details of ejecta from supernova (SN) explosions as well as those of the circumstellar medium (CSM) produced over the final evolutionary stages of massive stars. An obvious group of such young core-collapse SNRs is O-rich SNRs that show optical spectra featuring strong $\mathrm{O}$ and Ne lines, with lines from lighter elements (e.g., He) either weak or absent. Since the ejecta from a progenitor and the swept-up CSM in the O-rich SNRs are not completely mixed together yet, observation of the O-rich SNRs is promising to study the ejecta and CSM related to the explosion of a progenitor and its late-stage evolution.

G292.0+1.8 (MSH11-54), together with Cassiopeia A and Puppis A, forms a rare group of O-rich SNRs in the Galaxy. The O-rich nature of G292.0+1.8 was discovered by the optical detection of fast-moving O-rich and Ne-rich ejecta (Goss at al. 1979; Murdin \& Clark 1979). Recent wide-field observations of the optical [O III] line in G292.0+1.8 have revealed that the ejecta velocities range from $-1400 \mathrm{~km} \mathrm{~s}^{-1}$ to $+1700 \mathrm{~km} \mathrm{~s}^{-1}$ and also that distant ejecta knots are located primarily along the north-south direction (Ghavamian et al. 2005; Winkler \& Long 2006). The dynamical center of G292.0+1.8, based on the kinematics of 
the [O III] line emission from the ejecta, is roughly coincident with the geometrical center of the radio emission, but shows an apparent offset from its pulsar position discovered at the southeast (Winkler et al. 2009, see below). Ghavamian et al. (2009) reported that the mid-infrared (MIR) spectrum of the ejecta is dominated by ionic lines and a broad bump around $17 \mu \mathrm{m}$ using the Spitzer Space Telescope (Spitzer). They proposed that the latter is produced either by Polycyclic Aromatic Hydrocarbons (PAHs) along the line of sight or newly-formed dust within the ejecta. Besides there is also an equatorial MIR continuum component from the dust associated with the shocked CSM partly overlapping with the ejecta emission. A previous infrared (IR) study based on the data obtained with Infrared Astronomical Satellite $(I R A S)$, on the other hand, identified enhanced far-IR (FIR) emission in the southwestern part of the SNR, suggesting that the source has encountered adjacent clouds (Braun et al. 1986; Park et al. 2007).

In radio, G292.0+1.8 mainly consists of bright emission from a central pulsar wind nebula $(\mathrm{PWN})$ of $\sim 4^{\prime}$ (in diameter) and relatively fainter outer plateau emission of $\sim 9^{\prime}$ (Lockhart et al. 1977; Braun et al. 1986; Gaensler \& Wallace 2003). The plateau emission declines sharply outward, and there is no apparent surrounding radio shell structure. The pulsar PSR J1124-5916, which is located $\sim 46^{\prime \prime}$ from the dynamical center of the SNR in the southeast direction, was discovered by radio timing (Camilo et al. 2002), confirming the PWN nature of the central emission of the SNR. The pulsar and PWN were also identified in X-ray emission (Hughes et al. 2003), where the pulsar has a nearby jet and a torus of $\sim 5^{\prime \prime}$ scale (Park et al. 2007). The jet axis is slightly tilted to the northeast direction (Park et al. 2007). There are two clear differences between the soft and hard X-ray emission of G292.0+1.8. First, while the former is produced by shocked gas of normal composition distributed as equatorial filaments, indicating it is likely from CSM, the latter is dominated by bright metallic lines from the ejecta (Park et al. 2002, 2004). Next, the X-ray emission is harder in the northwestern part than the southeastern part, implying density variation or asymmetric SN explosion (Park et al. 2007). The distance to G292.0+1.8, on the other hand, was estimated to be $6.2 \mathrm{kpc}$ from H I absorption observations (Gaensler \& Wallace 2003).

In this paper we present an extensive IR study of G292.0+1.8 using multi-band imaging and spectroscopic data obtained with the $A K A R I$ space telescope (Murakami et al 2007). We describe the details of our $A K A R I$ observations and present the results of the observations in $\S 2$ and 3, respectively. In $\S 4$ we discuss IR emission in G292.0+1.8 from circumstellar dust with a comparison to model calculations of dust destruction by a SN shock. We also discuss the IR emission from the ejecta and we suggest that there is a negligible amount of dust associated with the ejecta. Then, we make a comparison to the results of Spitzer obserations. We finally discuss the SN explosion in G292.0+1.8 based on our AKARI observations 
followed by our conclusions in $\S 5$.

\section{Observations}

The AKARI multi-band imaging observations of G292.0+1.8 were carried out using its six near-IR (NIR) and MIR bands in the 2.7-26.5 $\mu \mathrm{m}$ range as well as four FIR bands in the 50-180 $\mu \mathrm{m}$ range on 2007 January 17 and 19. The NIR and MIR images were obtained with Infrared Camera (IRC) that has three simultaneously operating NIR, MIR-S, and MIR-L channels. The three channels have comparable field of views of $\sim 10^{\prime} \times 10^{\prime}$. The NIR and MIR-S channels share the same pointing direction, while that of the MIR$\mathrm{L}$ channel is $25^{\prime}$ away. The IRC observations were conducted in the two-filter mode that produced images of two bands for each channel (Onaka et al. 2007). The total on-source integration times were $178 \mathrm{~s}$ for the NIR (N3, N4) observations and $196 \mathrm{~s}$ for both the MIRS (S7, S11) and MIR-L (L15, L24) observations. The basic calibration and data reduction, including dark subtraction, linearity correction, distortion correction, flat fielding, image stacking, and absolute position determination were performed by the standard IRC Imaging Data Reduction Pipeline (version 20071017)1. Tables 1 and 2 present a journal of our $A K A R I$ observations, including the spectroscopic observations, and the basic parameters of the $A K A R I$ imaging bands, respectively.

The FIR images were obtained with Far-Infrared Surveyor (FIS) in two round-trip scans using the cross-scan shift mode (Kawada et al. 2007). The scan speed and length were $15^{\prime \prime} \mathrm{s}^{-1}$ and $240^{\prime \prime}$, respectively, creating images of $40^{\prime} \times 12^{\prime}$ size elongated along the scanning direction. All the four FIS band (N60, Wide-S, Wide-L, and N160) images were obtained simultaneously by a single observing run (Table11). The initial data calibration and reduction such as glitch detection, dark subtraction, flat fielding and flux calibration were processed with FIS Slow-Scan Toolkit (version 20070914)2, and the final image construction was performed with a refined sampling mechanism.

These IRC and FIS multi-band imaging observations were followed by NIR and MIR spectroscopic observations and L18W-band (13.9-25.6 $\mu \mathrm{m}$, combining both L15 and L24 bands) imaging observations carried out in 2007 July 20-22. The spectroscopic observations were conducted using four (NG, SG1, SG2, and LG2) grisms. Similar to the aforementioned imaging observations, the NG, SG1, and SG2 mode observations were conducted simulta-

\footnotetext{
${ }^{1}$ http://www.ir.isas.jaxa.jp/ASTRO-F/Observation/DataReduction/IRC/

${ }^{2}$ http://www.ir.isas.jaxa.jp/ASTRO-F/Observation/DataReduction/FIS/
} 
neously, while LG2 mode observations were done separately (Ohyama et al. 2007). Table 3 lists the characteristics of the $A K A R I$ spectroscopic observations. Spectra from the peaks of the equatorial emission and ejecta identified in the ratio between the L15- and L24-band images (L15/L24 hereafter) were obtained together with that from a reference background position at the southeastern part of the source (Table 4). The data calibration and reduction

were processed with IRC Spectroscopy Toolkit (version 20070913)3. The obtained flux was converted to the surface brightness based on the measured slit size. IRC spectroscopy is made at the slits located at the edge of the large imaging field-of-view (FoV). There is internal scattered light in the array of the MIR-S and the light diffusing from the imaging FoV affects SG1 and SG2 spectra to some extent. This effect has been corrected for according to the method given in Sakon et al. (2008). A similar scattered light is also recognized in LG2 spectra and has been corrected for in a similar way. In addition there is a contribution from the second order light from the edges of the imaging FoV in LG2 spectra, but we expect that it is not significant in the background-subtracted spectra.

These spectroscopic observations were simultaneously accompanied by supplemental short $(49 \mathrm{~s} \times 3)$ L18W-band imaging observations of a field $\sim 5^{\prime}$ away from the central slit position in the southeast direction in order to check the pointing of the satellite. This supplemental, short-exposure L18W-band image of the southeast, which fortuitously revealed interesting structures beyond the SNR boundary (see § 3.4), was combined with deep (442 s) L18W-band image of the source to generate a final larger-area map. The L18W-band imaging data were processed with the same procedures that we used for other imaging data sets as described above.

\section{Results}

\subsection{Multi-band Infrared Images of G292.0+1.8}

Figure 1 presents our $A K A R I$ IRC multi-band images of G292.0+1.8 together with the ATCA $20 \mathrm{~cm}$ radio continuum image (Gaensler \& Wallace 2003), Chandra X-ray (0.3$8 \mathrm{keV}$ ) image (Park et al. 2002), and the background-subtracted $A K A R I$ S11-band image (S11-S7; see below) for comparison. The $A K A R I$ IR emission associated with the SNR is most apparent in the L15- and L24-band images as two prominent features: first, there is a ring-like structure composed of two clumpy, narrow, and long filaments crossing the central part of the SNR along the east-west direction - we name this "Equatorial Ring" (ER);

\footnotetext{
${ }^{3}$ http://www.ir.isas.jaxa.jp/ASTRO-F/Observation/DataReduction/IRC/
} 
secondly, the outer boundary of the SNR appears as an almost-complete shell structure with its eastern part opened - we name this "Outer Elliptical Shell" (OES).

The radius of the $\mathrm{ER}$ is $\sim 3^{\prime}$ ( or $\sim 5 \mathrm{pc}$ ). Its southern filament is brighter than the northern one by a factor of $\sim 1.7$ on average and has the brightest clump at (RA, decl.) $=$ $\left(11^{\mathrm{h}} 24^{\mathrm{m}} 29^{\mathrm{s}} .4,-59^{\circ} 15^{\prime} 48^{\prime \prime}\right)$ close to the filament center. The southern filament also shows prominent X-ray and [O III] emission and has been called "equatorial bar" or "equatorial belt" (Park et al. 2002; Gonzalez \& Safi-Harb 2003; Ghavamian et al. 2005). The northern filament is $\sim 1^{\prime}$ away from the southern filament and both filaments are elongated roughly parallel to each other.

The OES shows an almost-complete ellipse of $\sim 7^{\prime} \times 6^{\prime}(\sim 12 \mathrm{pc} \times 10 \mathrm{pc})$ with its major axis aligning roughly with the plane of the ER. The center of the ellipse determined by elliptical fits weighted by the $24 \mu \mathrm{m}$ surface brightness of the OES is $\left(11^{\mathrm{h}} 24^{\mathrm{m}} 30^{\mathrm{s}} .9\right.$, $-59^{\circ} 15^{\prime} 21^{\prime \prime}$ ) located at the middle of two filaments of the ER. In the west the OES appears to be connected with the two filaments of the ER and its emission is enhanced there, especially at the southwest near the southern filament of the ER. In contrast the eastern part of the OES is open, and also the emission from the southern filament of the ER is truncated in the east before it reaches the boundary of the SNR.

In addition to the ER and OES, there appear to be at least two more clearly identifiable features in the $A K A R I$ images. First, the L15-band image shows a separate structure that extends $\sim 5^{\prime}$ southward from the ER on the southeastern side of the SNR. It is elongated vertically with the bright portion located $\sim 0.5^{\prime}$ below the southern equatorial filament. Next, there is faint emission extending beyond the northern and southeastern edges of the OES. This faint emission is contained within the outermost boundary of radio emission observed in G292.0+1.8 (Gaensler \& Wallace 2003).

As in Figure 1, G292.0+1.8 is not detected in the S7- and S11-band images: while some of the S11-band emission appears to arise from locations of strong L15- and L24-band emission, there is almost no corresponding emission in the S7-band image. This suggests that the S7-band emission is dominated by background emission, probably emission from PAHs in the line of sight interstellar medium (ISM). In the S11-S7 difference image (Figure 1) we subtract out scaled S7-band emission from the S11-band image and confirmed that the two filaments of the ER and southwestern part of the OES have appreciable S11-band emission. (Note that the three bright point-like sources close to the eastern end of the southern filament of the ER in the S11-S7 difference image are stellar sources.) The NIR N3- and N4-band images, on the other hand, show only stellar emission without any apparent feature brighter than $16 \mu \mathrm{Jy}$ at $3 \mu \mathrm{m}$ that might be associated with the SNR. 
Table 5 presents IR measurements of G292.0+1.8. In the Table, we list the fluxes and ratios of whole remnant area and IR-Ejecta region (see befow for IR-Ejecta). In addition, we also list the peak intensities and ratios of the ER, the southweastern OES, and the IR-Ejecta.

\subsection{Infrared Colors and Ejecta Identification}

Figure 2 (left), which is a three-color (7, 15 and $24 \mu \mathrm{m})$ AKARI MIR image of G292.0+1.8, shows that most of the prominent features have roughly similar MIR colors. One exception is the feature in the southeastern part of the SNR with significant excess in the shorter wavebands - we name this feature "IR-Ejecta" because it is believed to originate in shocked SN ejecta as we describe below. It is bright near the southern equatorial filament and stretching directly southward beyond the SNR boundary. This feature is clearly seen in Figure 2 (right) which shows the L15/L24 emission ratio. The ratio image was produced by dividing the L15-band image by the L24-band image after background subtraction. The background was estimated by fitting a slanted plane to the areas surrounding the SNR. The resulting background planes were almost flat, i.e., the brightness differences over the entire images were only $1.7 \%$ and $2.4 \%$ in L15 and L24, respectively. We applied a Gaussian convolution to the L15-band image in order to match the final spatial resolution to that of the L24-band image and masked out the pixels with small $\left(\leq 30.2 \mathrm{MJy} \mathrm{sr}^{-1}\right)$ L24-band intensities.

The resulting L15/L24 color image is significantly different from the original band images. In Figure 2, the L15/L24 ratio is almost uniform $(\sim 0.25)$, and there is no feature corresponding to the prominent ER and OES. The most notable feature is the IR-Ejecta, where L15/L24 ratio raises to $\sim 0.8$, which is in fact consistent with what we identified in the three-color image (left panel of Figure 22). It appears to be of a triangular shape with one of its vertices towards the direction to the SNR center. The southern vertex passes though the southern boundary of the OES and extends over the SNR boundary, while the northeastern vertex is located just above the ER. The region of high color ratio of the IREjecta covers a large portion of the southeastern area of the SNR and positionally coincides with the O- and Ne-dominant ejecta identified by previous optical and X-ray observations (Ghavamian et al. 2005; Winkler \& Long 2006; Park et al. 2002). There is also a wispy patch of emission around $\left(11^{\mathrm{h}} 24^{\mathrm{m}} 43^{\mathrm{s}} .4,-59^{\circ} 20^{\prime} 47^{\prime \prime}\right)$ far $\left(>1^{\prime}\right)$ beyond the SNR boundary, which has the color ratio similar to that of the IR-Ejecta. It, however, is not shown in the [O III] images (Winkler \& Long 2006). Besides the IR-Ejecta in the southeastern region, the northwestern region shows extended emission of the high color ratio, although it is not as significant as the southeastern region. Overall the two regions of the high color ratio are 
roughly symmetric with respect to the center of the OES.

Figure 3 compares the pixel values of the L24- and L15-band emission, where most of the points are distributed along the thick, solid line of a slope of 0.25. (Note that pixels of stars and pixels with small L24-band intensities are masked out.) If L24- and L15-band fluxes are from the same region and if they are both thermal dust emission, then the color ratio and the dust temperatures are related as

$$
\frac{I_{\nu}(15)}{I_{\nu}(24)}=\frac{\kappa_{\nu}(15) B_{\nu}(15, T)}{\kappa_{\nu}(24) B_{\nu}(24, T)},
$$

where $I_{\nu}(\lambda)$ is the surface brightness at $\lambda(\mu \mathrm{m})$ in $\mathrm{Jy} \mathrm{sr}^{-1}, B_{\nu}(T)$ is the Planck function, and $\kappa_{\nu}(\lambda)$ is the dust opacity in $\mathrm{cm}^{2} \mathrm{~g}^{-1}$. The slope of 0.25 corresponds to the dust temperature of $126 \mathrm{~K}$ for a mixture of carbonaceous and silicate interstellar grain of $R_{\mathrm{V}}=3.1$ (Draine) 2003). In Figure 3 there are two regions, where the data points show a high L15/L24 ratio compared to the thick, solid line: first, the thin line of the slope of 0.88 represents one group whose L24-band surface brightness is less than $\sim 33 \mathrm{MJy} \mathrm{sr}^{-1}$; secondly, there is another group of data points of higher L15-band emission whose L24-band surface brightness lies in the range of 33-40 $\mathrm{MJy} \mathrm{sr}^{-1}$. The inset in the lower-right corner of Figure 3 shows that the data points with high L15/L24 ratios are all from the IR-Ejecta as expected. The ones in the second group, i.e., ones with high $24 \mu \mathrm{m}$ surface brightness, are superposed on the ER and therefore their emission is partly from the swept-up CSM while the data points in the first group should represent the emission only from the ejecta. The slope of 0.88 corresponds to a dust color temperature of $240 \mathrm{~K}$; however the L15-band flux is largely from line emission at this position so that the physical dust temperature should be lower than this (see §3.4).

\subsection{Mid-Infrared Spectroscopy of Ejecta and Equatorial Peak}

Figure 4 shows the background-subtracted MIR grism spectra of the peak positions of the ER and the IR-Ejecta.4 (Note that the LG2 spectra at $>17 \mu \mathrm{m}$ were obtained from positions slightly shifted from those of the SG spectra of $5-14 \mu \mathrm{m}$ as we described in $\S 2$.) The spectrum from the IR-Ejecta peak clearly shows the [Ne II] line at $12.8 \mu \mathrm{m}$, which confirms the nature of the radiatively shocked ejecta. No Ar lines (i.e., [Ar II] at $7.0 \mu \mathrm{m}$ and [Ar III] at $9.0 \mu \mathrm{m})$ were detected, while neither [Ne III] line at $15.6 \mu \mathrm{m}$ nor [O IV] line at $25.9 \mu \mathrm{m}$ was covered. Table 6 lists the flux of the [Ne II] line and upper limits of the [Ar II]

\footnotetext{
${ }^{4}$ We only present the MIR spectra because NIR spectra were heavily contaminated by emission from nearby field stars.
} 
and [Ar III] lines. The flux of the [Ne II] line at $12.8 \mu \mathrm{m}$ is $2.8 \pm 2.5 \times 10^{-5} \mathrm{erg} \mathrm{cm}^{-2} \mathrm{~s}^{-1} \mathrm{sr}^{-1}$, corresponding to the surface brightness of $0.24 \pm 0.21 \mathrm{MJy} \mathrm{sr}^{-1}$ in the L15-band image. Given the L15-band surface brightness of 1.6 $\pm 0.1 \mathrm{MJy} \mathrm{sr}^{-1}, \sim 15 \%$ of the L15-band emission is due to the [Ne II] line emission. For the S11 band, the observed [Ne II] line emission flux corresponds to the S11-band surface brightness of $0.12 \pm 0.11 \mathrm{MJy} \mathrm{sr}^{-1}$. This is equivalent to $\sim 55 \%$ contribution to the total S11-band emission, which is much larger than the case of the L15-band emission. The difference is because there is no strong emission line other than the [Ne II] line in the S11 band while there is additional [Ne III] $15.5 \mu \mathrm{m}$ line in the L15 band (see the spectral responses in Figure 4). (There could be some dust emission too. See $\S$ 4.3.) On the other hand, the spectrum at the ER peak is dominated by the continuum emission, although the LG2 spectra have a lower signal to noise ratio.

\subsection{Large-scale Infrared Emission around G292.0+1.8}

Figure 5 presents a combined L18W-band image $(\S 2)$ covering both the SNR and an extended area in the southeast. Besides the features of the SNR that we already described in previous sections, there is a notable feature of the L18-band emission in the south. The elongated "Narrow tail" is located around $\left(11^{\mathrm{h}} 24^{\mathrm{m}} 45^{\mathrm{s}},-59^{\circ} 22^{\prime} 00^{\prime \prime}\right), \sim 7^{\prime}$ apart from the center of the SNR. It is close to the IR-Ejecta in the southeastern part of the SNR and its elongation direction is roughly towards the center of the SNR. Its extent is $\sim 1.5^{\prime}$ or 2.6 pc. In addition, there is faint, diffuse emission toward the south and southeast too. The emission toward the southeast appers distinct - it appears to protrude from the open portion of the OES having similar outer boundary with radio plateau at the east and south, but extends $\sim 7^{\prime}$ southeast beyond the radio boundary of the SNR. We consider that this "Wide tail" could be associated with the SNR. There is large, diffuse H II region superposed on G292.0+1.8 on the sky (RCW 54; Rodgers et al. 1960), but its $\mathrm{H} \alpha$ emission is elongated along the northeast-southwest direction without any emission corresponding to the Wide tail in the Southern H-Alpha Sky Survey Atlas (Gaustad et al. 2001). Therefore, the Wide tail is not associated with H II regions and its association with the SNR is likely.

Figure 6 presents the AKARI FIS FIR-band images of G292.0+1.8. The images cover the entire SNR with a scan direction of northeast to southwest. The FIR emission of the SNR associated with the ER and OES is clearly detected in the N60- and Wide-S-band images with strong concentration in the southwestern part, although fine details are difficult to see because of their low spatial resolutions. The N160- and Wide-L-band images are, however, clearly different from images of the other bands. They show an elongated feature extended in the northwest-southeast direction which shows no correlation with the emission 
associated with the SNR. Also its peak position is located outside the boundary of the SNR. These indicate that the N160- and Wide-L-band emission is not directly associated with G292.0+1.8, although it is possible that the enhanced brightness in the SW part of OES is due to the interaction of the remnant with this extended structure (cf. Braun et al. 1986). The bottom panels of Figure 6 present the background-subtracted N60- and Wide-S-band images. The background emission was estimated by calculating scaling factors between the N60- and N160-band images and also between the Wide-S- and N160-band images from the areas outside the SNR. Compared to the N60- and Wide-S-band images of the top panels, the background-subtracted images show more clearly the emission associated with the SNR, including the northern part of the OES that is not clear in the orignial images.

\section{Discussions}

\subsection{Destruction of Circumstellar Dust}

\subsubsection{Infrared Emission from Shocked Circumstellar Dust}

The ER and OES are the most prominent features in our $A K A R I$ multi-band IR images (Figures 1 and 6). The ER is composed of two filaments, where the southern one is brighter than the northern counterpart. The southern filament appears to be of the normal composition in soft X-rays (Park et al. 2002) without any apparent radial motion in the optical (Ghavamian et al. 2005), which led the authors to conclude that it is CSM from the progenitor of the SN in G292.0+1.8. We suggest that the northern filament, which has been clearly found by $A K A R I$ observations, could be part of the same structure based on its similar distribution to the southern filament. We note that the bright clump at $\left(11^{\mathrm{h}} 24^{\mathrm{m}} 46^{\mathrm{s}}\right.$, $-59^{\circ} 14^{\prime} 42^{\prime \prime}$ ) near the eastern end of the northern filament has a counterpart in the [O III] image of Ghavamian et al. (2005). Its velocity is near zero, which supports that the northern and southern filaments form a single structure. Furthermore, it is most clearly visible at the $-120 \mathrm{~km} \mathrm{~s}^{-1}$ frame of the Rutgers Fabry-Perot velocity scan images and absent at the $0 \mathrm{~km}$ $\mathrm{s}^{-1}$ frame where the southern filament is brightest (Figure 2 of Ghavamina et al. 2005). We interpret this velocity difference as an indication suggesting that the northern and southern filaments are parts of a tilted, expanding ring structure produced by the progenitor of the SN. The location of the center point of the OES at the middle of the two filaments also reconciles with the interpretation, given the CSM-nature of the OES (see below). We note that such a, but smaller, ring structure of the CSM was found in SN 1987A and also possibly in the Crab nebula (Bouchet et al. 2004; Green et al. 2004). The OES, on the other hand, is relatively fainter in X-rays than the ER (Park et al. 2002). The AKARI MIR bright- 
ness and color of the OES, however, are similar to those of the ER (Figure 2). Toward the southern filament of the ER, Ghavamian et al. (2005) reported the detection of the optical radiative lines produced by the partially radiative shocks starting to develop cooling zones. It implies that the X-ray emitting gas in the ER is cooler than that of the OES, while they have somewhat similar dust properties. And there is a possibility that the ER in S11-S7 difference image also contains the [Ne II] line emission produced in the CSM region where the shock has started to cool down to $<100,000 \mathrm{~K}$. However, its contribution might be small, because we have not detected the [Ne II] $12.8 \mu \mathrm{m}$ line at the ER.

In addition to the ER and OES, there are faint MIR emissions beyond the northern and southeastern edges of the OES (Figure 2). They have sharp outer boundaries, representing the current locations of the SN blast wave. Faint X-ray emission was detected in those areas too (Park et al. 2002). It is possible that the SN blast wave has overtaken the OES and produced those emission features while propagating into a more diffuse medium. On the other hand, it is also possible that the remnant has a front-back asymmetry and they are just projected boundaries of the more-extended shell. In any case, their asymmetric spatial distribution suggests that either the ambient density distribution or the mass ejection from progenitor was asymmetric.

As in Figure 4, the MIR emission of G292.0+1.8 is dominated by continuum emission, not by line emission. This implies that the IR emission is from shock-heated dust grains in the CSM. Figure 7 presents the spectral energy distribution (SED) of the SNR in Table 5 , We applied modified blackbody fits composed of two dust components to the observed SED in order to obtain the best-fit dust temperatures. The dust model based on a mixture of carbonaceous and silicate interstellar grain of $R_{\mathrm{V}}=3.1$ (Draine 2003) gave dust temperatures of $\sim 103 \mathrm{~K}$ (warm dust) and $\gtrsim 47 \mathrm{~K}$ (cold dust), corresponding to the mass of $4.5 \pm 0.9$ $\times 10^{-4} \mathrm{M}_{\odot}$ and $\lesssim 4.8 \times 10^{-2} \mathrm{M}_{\odot}$, respectively. (Note that the lower limit of the cold dust temperature comes from the upper limit of the flux at $140 \mu \mathrm{m}$.) For the case of the dust model based on the graphite and silicate grain of 0.001 to $0.1 \mu \mathrm{m}$ size (Draine \& Lee 1984; Laor \& Draine 1993), the derived total mass is in the range of $(1.0-3.4) \times 10^{-2} \mathrm{M}_{\odot}$, comparable to the total mass obtained for the former model. The derived dust mass corresponds to the dust-to-gas ratio of $\lesssim 1.6 \times 10^{-3}$, if we use the $30.5 \mathrm{M}_{\odot}$ swept-up mass (at distance of $6.2 \mathrm{kpc}$ ) of the gas in the CSM obtained in X-ray observations (Gonzalez \& Safi-Harb 2003). This is lower than the ratio $6.2 \times 10^{-3}$ found in the local ISM (Zubko et al. 2004). The low dust-to-gas ratios in the swept-up materials by the shock destruction were also obtained with Spitzer observations on the core-collapse SNRs in the Large Magellanic Cloud (Williams et al. 2006) and the Kepler (Blair et al. 2007). According to our result, $\gtrsim 75 \%$ of the dust in G292.0+1.8 might have been destroyed by the SN shock, or the initial dustto-gas ratio surrounding G292.0+1.8 might be lower than the local value. It is comparable 
to the fraction derived in other SNRs, such as $64 \%$ in Cas A, (Dwek et al. 1987) and $78 \%$ in Kepler (Blair et al. 2007).

\subsubsection{Model Calculations of Shock-heated Dust Emission}

We perform model simulations for the destruction of dust by SN shock waves and the thermal emission from shock-processed dust. The physical processes of dust in shocks have been so far discussed in many works (e.g., Tielens et al. 1994; Vancura et al. 1994; Dwek et al. 1996; Jones 2004). Once the circumstellar dust grains are swept up by the blast wave, they acquire high velocities relative to the gas and are eroded by kinetic and/or thermal sputtering in the shock-heated gas. Dust grains are also heated by collisions with energetic electrons in the hot gas and radiate thermal emission at IR wavelengths. Dynamics, erosion, and temperature of dust depend on the temperature and density of the gas as well as the chemical composition and size of dust grains.

We adopt the model of dust destruction calculation by Nozawa et al. (2006), in which the motion, destruction, and heating of dust in the shocked gas are treated in a self-consistent manner by following the time evolution of the temperature and density of the gas for the spherically symmetric shock wave. As the initial condition of the SN ejecta we consider the freely expanding ejecta with the velocity profile of $V=V_{\text {eje }}\left(r / R_{\text {eje }}\right)$ and the density profile of $\rho=\rho_{\text {core }}$ at $r \leq R_{\text {core }}$ and $\rho=\rho_{\text {core }}\left(r / R_{\text {eje }}\right)^{-\alpha}$ at $r>R_{\text {core, }}$ where $V_{\text {eje }}$ and $R_{\text {eje }}$ are the velocity and radius of the outermost ejecta, respectively. Taking the kinetic energy of $10^{51}$ ergs and the ejecta mass of $8 M_{\odot}$ (Gaensler \& Wallace 2003) for the density profile of core-collapse SNR of $R_{\text {core }}=0.3 R_{\text {eje }}$ and $\alpha=12$ (e.g., Chevalier 1982; Matzner \& McKee 1999; Pittard et al. 2001), we obtain $\rho_{\text {core }}=1.4 \times 10^{-19} \mathrm{~g} \mathrm{~cm}^{-3}, V_{\text {eje }}=1.3 \times 10^{9} \mathrm{~cm} \mathrm{~s}^{-1}$, and $R_{\text {eje }}=4.2 \times 10^{17} \mathrm{~cm}$ at $10 \mathrm{yrs}$ after explosion, when the simulations are started. Since most of the dust grains swept up by the forward shock during the later epoch of the evolution, the calculation results are not sensitive to the ejecta structure.

For the ambient medium we consider the constant hydrogen number density of $n_{\mathrm{H}, 0}=$ $0.1,0.5,1$, and $10 \mathrm{~cm}^{-3}$. The circumstellar dust is assumed to be amorphous carbon or silicate (forestrite) with the power-law size distributions $\left(\propto a^{-3.5}\right)$ ranging from $a=0.001$ $\mu \mathrm{m}$ to $a=0.5 \mu \mathrm{m}$ (Mathis et al. 1977). The optical constants are taken from Edo (1983) and Semenov et al. (2003). Based on the time evolution of the size distribution and temperature of dust given by the simulation and the assumption of the dust grains being in thermal equilibrium, we calculate the IR SED by thermal emission from the shock-heated dust. The detailed description for calculating the IR SED from shocked dust will be given elsewhere (T. Nozawa et al. 2009 in preparation). 
Figure 8 compares the observed fluxes of G292.0+1.8 with the calculated IR SEDs at 3,000 yrs for $n_{\mathrm{H}, 0}=0.1,0.5,1$, and $10 \mathrm{~cm}^{-3}$. We present the results with the initial dust-togas mass ratio to best reproduce parts of the observed SED, for amorphous carbon (Figure 8a) and silicate (Figure 8b). The typical temperatures of dust are 35-55, 45-65, 50-70, and 60$80 \mathrm{~K}$ for $n_{\mathrm{H}, 0}=0.1,0.5,1$, and $10 \mathrm{~cm}^{-3}$, respectively, and the resulting dust masses are in the range of $(0.3-5) \times 10^{-2} M_{\odot}$ for carbon and $(0.4-8) \times 10^{-2} M_{\odot}$ for silicate with the higher values for lower ambient density (thus lower temperature of dust). It can be seen that the results of the silicate grains with $n_{\mathrm{H}, 0}=0.5 \mathrm{~cm}^{-3}$, which coincides with the density estimated from X-ray observations (Gonzalez \& Safi-Harb 2003), can reasonably reproduce the overall shape of the IR SED for G292.0+1.8. In this case, the mass of grains radiating IR emission is $2.9 \times 10^{-2} M_{\odot}$. Note that the initial dust-to-gas mass ratio corresponding this result is $1 \times 10^{-3}$, which is smaller than that in the local Galaxy. If the IR emission originates in the swept-up materials containing the mass-loss wind of RSG with the solar metallicity, the condensation of silicate is expected, and, according to our results, its condensation efficiency could be low.

It should be noted here that the simulation results for $n_{\mathrm{H}, 0}<10 \mathrm{~cm}^{-3}$ significantly underestimates the flux at short $(11 \mu \mathrm{m})$ wavelength. However, the SED at shorter wavelengths could be resolved by including the effect of a stochastic heating of small grains; stochastically heated dust produces more emission at shorter wavelengths than the dust with equilibrium temperature, and it may also allow acceptable fits for lower initial density and/or different size distribution of dust than the current best fit. Alternatively, this disagreement may be caused by the difference in the assumed composition of dust. To gain deeper insights into the properties of dust in G292.0+1.8, we need further investigations by taking account of the stochastic heating and changing the composition and size distribution of dust as well as the density profile in the ambient medium.

\subsection{Infrared Emission from Supernova Ejecta}

\subsubsection{Ejecta Neon Line Emission}

We have detected MIR emission associated with the SN ejecta: IR-Ejecta. It is prominent in the 15/24 $\mu \mathrm{m}$ ratio image by its high L15/L24 ratio (Figure 2), but is marginally seen in the total intensity maps of the MIR $(11-24 \mu \mathrm{m})$ and FIR $(65-90 \mu \mathrm{m})$ too. The emitting area coincides with the fast-moving $\left(<1,500 \mathrm{~km} \mathrm{~s}^{-1}\right)$, O-rich SN ejecta: the triangle-shaped bright portion near the equatorial filament was called "spur" and the extension to the south was called "streamers" by Ghavamian et al. (2005). In the high-resolution [O III] 5007 image, the spur is crescent-shaped with a sharp boundary toward the SNR center, while the 
streamer appears to be composed of clumps embedded in diffuse emission. Recent measurement of their proper motions showed that they are expanding systematically from a point near the geometrical center of the OES (Winkler et al. 2009, see $\S 4.4$ too).

According to our spectroscopic result, the IR-Ejecta shows [Ne II] $12.8 \mu \mathrm{m}$ emission but no $[\mathrm{Ar}$ II $] 7.0$ or [Ar III] $9.0 \mu \mathrm{m}$ emission. Note that the latter lines from Ar ions were also detected at the metal-rich ejecta in young SNRs (Douvion et al. 2001; Smith et al. 2009; Williams et al. 2008). The lack of IR line emission from Ar ions in this area is consistent with the results of optical or X-ray studies which showed that there is no line emission from elements heavier than S in this area (Ghavamian et al. 2005 and references therein; Park et al. 2002). The absence of Ar lines supports the claim by Ghavamian et al. (2005) that we are not seeing the ejecta in the inner-most region accelerated by pulsar wind nebula but seeing the He-burning-synthesized, O-rich ejecta swept-up by reverse shock.

The IR-Ejecta shows high L15/L24 ratio, i.e., L15/L24=0.88 compared to 0.25 of the shocked CSM. The high L15/L24 ratio is at least partly due to Ne lines. (There is [O IV] $25.9 \mu \mathrm{m}$ line in the $\mathrm{L} 24$ band, but it is weak, i.e., $\sim 20 \%$ of the $[\mathrm{Ne} \mathrm{II}]+[\mathrm{Ne} \mathrm{III}]$ lines. See $\S$ 4.3.) According to our estimation, [Ne II] $12.8 \mu \mathrm{m}$ line contributes $\sim 15 \%$ to the L15 flux. In the L15 band, there is another strong Ne line, [Ne III] $15.6 \mu \mathrm{m}$ line. We may estimate the possible contribution of [Ne III] $15.6 \mu \mathrm{m}$ line in G292.0+1.8 as follows.

The strength of a forbidden Ne line is given by $I_{21}=\left(h \nu_{21} / 4 \pi\right) N_{2} A_{21}$ where $\nu_{21}$ is the frequency of the line, $N_{2}$ is the column density of $\mathrm{Ne}^{+}$or $\mathrm{Ne}^{++}$ions in the upper state along the line of sight, and $A_{21}$ is the Einstein coefficient, so that the [Ne III] $15.6 \mu \mathrm{m} /[\mathrm{Ne}$ II] 12.8 $\mu \mathrm{m}$ ratio is given by

$$
\frac{I_{15.6 \mu m}}{I_{12.8 \mu m}}=0.57 \frac{N\left(\mathrm{Ne}^{++}\right)}{N\left(\mathrm{Ne}^{+}\right)} \frac{f\left(\mathrm{Ne}^{++},{ }^{3} P_{1}\right)}{f\left(\mathrm{Ne}^{+},{ }^{2} P_{1 / 2}\right)},
$$

where $N\left(\mathrm{Ne}^{++}\right)$and $N\left(\mathrm{Ne}^{+}\right)$are column densities of $\mathrm{Ne}^{++}$and $\mathrm{Ne}^{+}$ions, $f\left(\mathrm{Ne}^{++},{ }^{3} P_{1}\right)$ and $f\left(\mathrm{Ne}^{+},{ }^{2} P_{1 / 2}\right)$ are the fractions of $\mathrm{Ne}^{++}$and $\mathrm{Ne}^{+}$ions in the upper states of the corresponding emission lines (see Glassgold et al. 2007 for atomic parameters of these lines). Assuming statistical equilibrium, the last factor, $f\left(\mathrm{Ne}^{++}, 3{ }^{3}\right) / f\left(\mathrm{Ne}^{+},{ }^{2} P_{1 / 2}\right)$, can be easily calculated (e.g., Glassgold at al. 2007), and varies from 1 to 2 as the electron density increases from a lowdensity limit to densities much higher than the critical density $\left(=(4-6) \times 10^{5} \mathrm{~cm}^{-3}\right.$ at 10,000 $\mathrm{K})$. Therefore, the intensity ratio $I_{15.6 \mu \mathrm{m}} / I_{12.8 \mu \mathrm{m}}$ is $\lesssim 1$ unless $N\left(\mathrm{Ne}^{++}\right) \gg N\left(\mathrm{Ne}^{+}\right)$, which happens at temperatures $\gtrsim 10^{5} \mathrm{~K}$ in collisional equilibrium (e.g., Allen \& Dupree 1969 ). Ghavamian et al. (2005) suggested that ejecta undergoes radiative shocks with a velocity of 50-200 $\mathrm{km} \mathrm{s}^{-1}$ from the [O III] 5007 line widths. The temperature of shocked ejecta gas immediately behind the shock of velocity $v_{s}$ should be high, i.e., $4.5 \times 10^{6}\left(v_{s} / 100 \mathrm{kms}^{-1}\right)^{2} \mathrm{~K}$, assuming pure neon preshock gas, but since the shocked gas element cools fast due to en- 
hanced heavy elements and the column density of the gas at $T$ might vary roughly with $1 / T$, we expect $N\left(\mathrm{Ne}^{++}\right)<N\left(\mathrm{Ne}^{+}\right)$and therefore $I_{15.6 \mu m} / I_{12.8 \mu m} \lesssim 1$. Toward the $\mathrm{SN}$ ejecta in young core-collapse SNRs such as Crab, Cas A, the SNR B0540-69.3 in the LMC, or the SNR 1E0102-72.3 in the SMC, the [Ne III]15.6 $\mu \mathrm{m} /[\mathrm{Ne}$ II] $12.8 \mu \mathrm{m}$ ratio varies from 0.3 to $\sim 1$ too (Douvion et al. 2001; Temin et al. 2006; Smith et al. 2009; Williams et al. 2008; Rho et al. 2009). We may conclude that the contribution of the [Ne III] $15.6 \mu \mathrm{m}$ line to the L15 band should be at most comparable to that of the [Ne II] $12.8 \mu \mathrm{m}$ line unless the shock is truncated at relatively high temperatures.

\subsubsection{Dust Emission Associated with Ejecta}

We have derived IR fluxes associated with the ejecta and the results are summarized in Table 5. It is difficult to extract the ejecta emission in IR observations, although our results unveiled the ejecta region unambiguously. The IR-Ejecta is clearly divided into two regions (Figures 2 and 3); one that coincides with the ejecta-only region and has a tight correlation in the L15 vs L24 plot and the other superposed on the ER and has a loose correlation in the L15 vs L24 plot. These IR-Ejecta regions are part of the structure defined as the [O III] "Spur" by Ghavamian et al. (2005), with the ejecta-only region being the bottom of the Spur, and the ejecta superimposed on the ER being the top portion of the Spur. We measure the flux at the ejecta-only region masking the emission from stars. The area of the ejecta-only region is more than four times larger than the superposed ejecta region and it contains most of the flux. In order to remove the background emission, we subtract the average of nearby $\left(\sim 2^{\prime}\right)$ low intensity region.

The $A K A R I$ ejecta spectrum in Figure 4 shows that the 11 and $15 \mu \mathrm{m}$ fluxes are not from dust continuum. We believe that the S11-band is mostly dominated by line emission, while the L15-band needs an additional component (see $\S 3.3$ ). In the next paragraph, we show that some of the L15 flux is due to a bump at 15-25 $\mu \mathrm{m}$. On the other hand, FIR emission is certainly dominated by dust continuum. The L24-band contains the [O IV] $25.9 \mu \mathrm{m}$ line emission, whose wavelength is not covered with our spectroscopic observations. Its contribution in L24-band is expected to be less than $20 \%$ in our estimation using the [O IV] $25.9 \mu \mathrm{m}$ line intensity in Ghavamian et al. (2009). At first, we fit $24-140 \mu \mathrm{m}$ band fluxes using a modified-blackbody for silicate and graphite grain models of $0.001-0.1 \mu \mathrm{m}$ size (Draine \& Lee 1984; Laor \& Draine 1993). The best fits give temperature of $64-88 \mathrm{~K}$ and dust mass of $2.0-8.2 \times 10^{-5} \mathrm{M}_{\odot}$. If we exclude the L24-band data point, the temperature drops to $44-52 \mathrm{~K}$ and the dust mass increases by a factor of 5 . This IR-emitting dust mass is much smaller $\left(<4 \times 10^{-4}\right)$ than the theoretically predicted dust mass $\left(0.1-1 \mathrm{M}_{\odot}\right)$ to be 
formed in the core-collapse SN explosion (e.g., Nozawa et al. 2003).

\subsection{Comparison with Spitzer Spectroscopic Results}

Recently the results from MIR observations of G292.0+1.8 with the Spitzer satellite have been published (Ghavamian et al. 2009) based on low-resolution spectroscopy of the ejecta and the southern filament of the ER where the ejecta emission is superimposed on the emission from the CSM. The former is located $\sim 0^{\prime} .5$ north from our IR-Ejecta slit position, while the latter, which has a high L15/L24 ratio, is $\sim 1^{\prime}$ apart in the northeast direction. Their ejecta spectrum shows strong emission from the [Ne II] $12.8 \mu \mathrm{m}$, [Ne III] $15.6 \mu \mathrm{m}$, [O IV] $25.9 \mu \mathrm{m}$ lines and relatively weak [Ne V] $24.4 \mu \mathrm{m}$ and [Ne III] $36.0 \mu \mathrm{m}$ lines, but no lines of $\mathrm{Mg}, \mathrm{Si}, \mathrm{S}, \mathrm{Ar}$ or Fe are identified. (Note that the $\mathrm{Si}$ and $\mathrm{S}$ lines in their spectrum are not from the ejecta but from the background.) The observed ratio of [Ne II] $12.8 \mu \mathrm{m}$ to [Ne III] $15.6 \mu \mathrm{m}$ is 2.1-2.7. The non-detection of Ar lines and the observed ratio of $\mathrm{Ne}$ lines are consistent with our results and the prediction in $\S 4.2$. The observed flux of [O IV] $25.9 \mu \mathrm{m}$ line is $\sim 20 \%$ of the [Ne II] plus [Ne III] line fluxes (Ghavamian et al. 2009). If the AKARI L15 and L24 fluxes are entirely due to lines, we estimate that the L15/L24 ratio should be 2.3-2.6 using the line fluxes in Ghavamian et al. (2009). (It becomes higher if we consider the 15-25 $\mu \mathrm{m}$ bump in the next paragraph.) The observd ratio, however, is 0.25 for the ER and OES and 0.88 for the ejecta. Therefore, the contribution of [O IV] $25.9 \mu \mathrm{m}$ line flux to the observed L24-band flux should be small, particularly toward the ER where $I_{\nu}(24) / I_{\nu}(15) \approx 5(\S 3.2)$. We, however, note that the Spitzer IRS spectrum toward the ejecta in Ghavamian et al. (2009) does not show any obvious dust continuum emission between 24 and $36 \mu \mathrm{m}$. We consider that it could be because there was dust continuum emission in the IRS background. In the AKARI $24 \mu \mathrm{m}$ image, there is faint filament coincident with the IRS background region (LL1 Sky in Ghavamian et al. 2009). This filament is associated with the OES and its $24 \mu \mathrm{m}$ emission might be dominated by dust continuum, so that, by subtracting its spectrum from the ejecta spectrum, the continuum feature could have been removed.

An interesting feature in the Spitzer ejecta spectrum is a weak 15-25 $\mu \mathrm{m}$ bump, which was suggested to be produced by newly-formed dust or swept-up PAHs along the line of sight. First of all, this bump explains the high L15/L24 ratio at the ejecta region described in $\S$ 4.2.1. The peak of the bump feature appears in the L15 band. Therefore, it contributes mainly to the AKARI $15 \mu \mathrm{m}$ band. Secondly, our AKARI observations show that the areas with a high L15/L24 ratio are coincident with those of the optical O-rich ejecta region. This supports the interpretation that the bump feature is related to the newly-formed dust in 
association with the SN ejecta, not to the swept-up PAHs.

According to Ghavamian et al. (2009), the Spitzer spectrum from the southern filament of the ER is consistent with the emission from two dust components: a warm (or hot) component of $\approx 114 \mathrm{~K}$ and a cold component of $\approx 35 \mathrm{~K}$. The temperature of the warm dust is higher than what we estimated with the $A K A R I$ data by $\sim 10 \mathrm{~K}$, while that of the cold dust is lower than the $A K A R I$ temperature by a comparable amount. It is possible that the presence of the bump feature in the Spitzer spectrum resulted in a higher temperature

for the warm dust. For the cold dust temperature, as explained in Ghavamian et al. (2009), the absence of the longer wavelength $(>30 \mu \mathrm{m})$ data in the Spitzer spectrum is likely the primary reason of the lower temperature compared to the $A K A R I$ results. IR spectroscopic observations covering a broad band are necessary to clearly resolve the issues.

\subsection{Supernova Explosion in G292.0+1.8}

\subsubsection{Circumstellar Shell and the Explosion Location}

One interesting result that we obtained in this study is the difference $\left(\sim 42^{\prime \prime} \simeq 1 \mathrm{pc}\right)$ between the center of the CSM and the dynamical center of the ejecta (Figure 2). The former corresponds to the center of the ER and OES that we determined with the AKARI results; the latter was determined by the distribution of the O-rich ejecta in the optical, which is close to the center of the SNR in the radio emission. In addition, the position of the pulsar is different from the both positions - it is shifted in the southeast direction from the dynamical center by $\sim 46^{\prime \prime}$.

One possibility is that the progenitor star was at the center of OES during its RSG phase but exploded at the position of the dynamical center of optical knots. This is possible because the RSG wind could be confined by external pressure while the central star is moving. According to Chevalier (2005), the RSG wind from a $25-35 \mathrm{M}_{\odot}$ star, which explodes as SN IIL/b, would be pressure confined while its outer radius becomes $\gtrsim 5 \mathrm{pc}$. The radius of OES (6 pc) is comparable to what the theory predicts. If the progenitor star was moving at $\sim 10 \mathrm{~km} \mathrm{~s}^{-1}$ and exploded after $\sim 10^{5}$ yrs of the pressure confinement of the shell, then the explosion center would be close to the dynamical center of optical knots.

Another possibility is that the SN exploded at the center of ER and OES, not at the dynamical center of the ejecta or the center of the radio emission. This is motivated by the fact that the OES shows a very well-defined shell structure surrounding the SNR, thereby the center of the OES may pinpoint to the real location of the progenitor, which later exploded as a SN. On the other hand, the centers of the radio emission and the O-rich ejecta motion 
could have been weighted toward the southeast: Firstly, the geometrical center of the radio nebula could be significantly weighted toward the southeast because of the existence of the bright PWN. Secondly, the dynamical center of optical knots is also weighted to the southeast because the bright optical knots in the southeastern area are moving relatively slowly and the center is derived using an assumption of an unhindered constant velocity since the explosion (Winkler et al. 2009).

If the SN explosion in G292.0+1.8 indeed occurred at the center of the OES, then the tangential velocity of the pulsar needs to be $\sim 1,000 \mathrm{~km} \mathrm{~s}^{-1}$. Although 1,000 $\mathrm{km} \mathrm{s}^{-1}$ is somewhat large as a pulsar kick velocity, it is still within the acceptable range (e.g., Ng \& Romani 2007), and the pulsar in another O-rich SNR Puppis A may also have such a high velocity (Hui \& Becker 2006).

\subsubsection{Ejecta Distribution and Explosion Asymmetry}

The optical and X-ray studies of the SN ejecta in G292.0+1.8 have shown that their spatial distribution and physical properties are not symmetric, including the northwestsoutheast concentration of the $\mathrm{O}$ and Ne ejecta, and the distinctive difference of the X-ray plasma temperature between the northwestern and southeastern areas (Park et al. 2002; Ghavamian et al. 2005; Park et al. 2007). Also the ejecta in the northern and southern boundaries move faster than those in the eastern and western areas (Winkler et al. 2009). It is worth to note that the pulsar jet axis is also along the northeast-southwest direction (Park et al. 2007).

Our $A K A R I$ result on the Ne-line emitting ejecta material identified by their high L15/L24 ratio is consistent with the spatial distribution seen in [O III] 5007: most of the $\mathrm{Ne}^{+} / \mathrm{Ne}^{++}$ions are distributed in the southeastern area called spur and streamers, several isolated ones coincide with the optical knots, and the other group of Ne-rich knots is distributed in the northwestern area and coincides with [O III] optical emission knots (fast-moving knots, or FMKs, as reported by Ghavamian et al. (2005) and Winkler \& Long $(2006))$. The northwest ejecta is less obvious in optical but it is easily recognized in the X-ray image of ionized $\mathrm{O}$ and Ne elements (Park et al. 2002). This type of bipolar ejecta distribution is also found in other young core-collapse SNRs, e.g., Cas A and G11.2-0.3 (Smith et al. 2009; Koo et al. 2007; Moon et al. 2009). Cas A shows a similar Ne ejecta distribution, which is almost perpendicular to the well-known narrow northeast-southwest jet, but is roughly aligned to the bipolar ionic ejecta, which sometimes is suggested be the major direction of explosion (Hwang et al. 2004; Wheeler et al. 2008; Smith et al. 2009). In G11.2-0.3, which is the remnant of the historical SN AD 386, the iron ejecta is found to be 
distributed mainly along northwest-southeast direction (Koo et al. 2007; Moon et al. 2009).

The symmetry axis in the spatial and kinematical distribution of ejecta in G292.0+1.8, therefore, is either along northwest-southeast or north-south, which is perpendicular to the plane of the ER. We consider that either the explosion was asymmetric and/or the CS wind was denser in the equatorial plane so that the ejecta expanding in this plane was slowed down more compared to those expanding to the other directions.

An interesting feature is the Narrow tail in the wide L18W-band image that extends from the end of the streamers to outside the remnant. A possible explanation is that the Narrow tail is a part of the O-rich streamer. Note that the Narrow tail is connected to the streamers by a southern patch of emission near the boundary of L15-band image. The distance from the center to the end of the Narrow tail is $7^{\prime}(13 \mathrm{pc})$, which is $\sim 2^{\prime}(3 \mathrm{pc})$ farther than the outermost O-rich clump in this area (Winkler et al. 2009). It, however, does not has an optical counterpart in the [O III] image (Winkler et al. 2009). If we assume a constant velocity and adopt an age of 3,000 yrs (Ghavamian et al. 2005; Winkler et al. 2009), the transverse velocity of the Narrow tail is $4,000 \mathrm{~km} \mathrm{~s}^{-1}$. This gives a possibility that its radial velocity is also very large, beyond the velocity range of previous optical narrow-band imaging observations (e.g., $\sim 2,000 \mathrm{~km} \mathrm{~s}^{-1}$ in Winkler \& Long 2006). An alternative explanation is that the Narrow tail is a reradiated IR light echo similar to the echoes identified in Cas A (Krause et al. 2005; Dwek \& Arendt 2008). The lack of counterpart in the optical [O III] and X-ray images, together with the faint feature in the FIR images (Figure 6), could be consistent with the continuum origin of the IR emission. In case of a light echo, the location of the echo can be derived from the geometrical equation of ellipse whose two focuses are the SN and the observer (e.g., Couderd 1939; Dwek \& Arendt 2008). Applying a projected radius of $13 \mathrm{pc}$ and a time delay of 3,000 yrs, we obtain a location of the IR echo at 450 pc behind G292.0+1.8 and its angular offset of $\sim 2^{\circ}$ from the line of sight. It appears that this alignment is too tight at a first glance, but it can be a selection effect caused by our limited imaging area. For example, Krause et al. (2005) discovered IR echoes along the scan direction in Cas A for the first time, but Dwek \& Arendt (2008) reported that the echoes were distributed in many positions in large $\left(\sim 2^{\circ}\right)$ area. More observations are definitely necessary to inspect the nature of the Narrow tail.

On the other hand, the sharp boundary of the SN blast wave was detected only toward the north and southwest (Figure 2). The absence of SN blast wave in the other directions implies that either the shock is trapped within the shell because that part of the shell has a larger column density or the SN blast wave has propagated far beyond because the ambient density is lower toward that direction. There is no indication in the $A K A R I$ images that the OES is denser, where the SN blast wave is missing. Instead those parts are fainter in the 
FIR images which indicates a lower column density. In this regard, the faint MIR emission that extends far beyond the bright shell to the southeast (Figure 51) is interesting because if it is part of the SNR, it indicates that the SN blast wave has propagated much further out toward this direction probably due to the lower ambient density. A deep radio observation could reveal faint features associated with this IR structure.

\section{Conclusions}

We have presented the NIR to FIR imaging and MIR spectroscopic observations of the O-rich SNR G292.0+1.8 using the IRC and FIS instruments aboard $A K A R I$ satellite. The almost continuous multiband imaging capability of $A K A R I$ covering wide IR wavelengths together its wide field of view enabled us to clearly see the distinct IR emission from the entire SNR and to derive its IR charactersitics. We derive the physical parameters of IRemitting dust grains in the swept-up circumstellar medium and compare the result with the model calculations of dust destruction by a SN shock. The overall shape of the observed SED can be explained by a simple model using characteristic SNR parameters with a bit lower initial dust-to-gas ratio. At $11 \mu \mathrm{m}$, the model flux is significantly smaller, which may indicate the importance of stochastic heating. We have not detected any signficant amount of freshly-formed dust associated with the SN ejecta.

The AKARI results in this paper give new insights into the explosion dynamics of G292.0+1.8. We have discovered an almost symmetric IR shell probably produced by the circumstellar wind from the progenitor star in the RSG phase. Its center is significantly offset from the previously suggested explosion centers. We consider that either OES represents the circumstellar shell pressure-confined by external medium or the SN exploded close to the center of the OES. In the latter case, the pulsar in G292.0+1.8 may be traveling at a speed of $\sim 1,000 \mathrm{~km} \mathrm{~s}^{-1}$. At the same time, the ejecta distribution is unveiled by their high 15 to $24 \mu \mathrm{m}$ ratio. The ejecta are mainly distributed along the northwest-southeast direction. This symmetric pre-supernova structure and asymmetric ejecta distribution appear to be rather common in the remnants of SN IIL/b, which suffer strong mass-loss like Cas A (Hines et al. 2004; Smith et al. 2009). There is also a Narrow tail outside the SNR shell, which might be similar to the feature observed in Cas A. A detailed study is necessary to understand the nature of this feature.

We suggest that multi-band IR imaging observations are powerful tools to explore both the ejecta and CSM emission in young core-collapse SNRs. Especially the 15 and $24 \mu \mathrm{m}$ images are useful to reveal the detailed structure of IR features, which leads to better understanding of environments of the progenitor and the SN explosion. 
This work is based on observations with $A K A R I$, a JAXA project with the participation of ESA. We wish to thank all the members of the $A K A R I$ project. We also thank $\mathrm{B}$. Gaensler for providing the ATCA $20 \mathrm{~cm}$ image and S. Park for providing the Chandra Xray image. This work was supported by the Korea Research Foundation Grant funded by the Korean Government (KRF-2008-357-C00052) and the Korea Science and Engineering Foundation (R01-2007-000-20336-0). This work was also supported in part by a Grant-inAid for Scientific Research for the Japan Society of Promotion of Science (18204014). T.N. has been supported in part by World Premier International Research Center Initiative (WPI Initiative), MEXT, Japan, and by the Grant-in-Aid for Scientific Research of the Japan Society for the Promotion of Science (19740094).

Facility: Akari

\section{REFERENCES}

Allen, J. W., \& Dupree, A. K. 1969, ApJ, 155, 27

Blair, W. P., Ghavamian, P., Long, K. S., Williams, B. J., Borkowski, K. J., Reynolds, S. P., \& Sankrit, R. 2007, ApJ, 662, 998

Bouchet, P., De Buizer, J. M., Suntzeff, N. B., Danziger, I. J., Hayward, T. L., Telesco, C. M., \& Packham, C. 2004, ApJ, 611, 394

Braun, R., Goss, W., M., Caswell, J. L., \& Roger, R. S. 1986, A\&A, 162, 259

Camilo, F., Manchester, R. N., Gaensler, B. M., Lorimer, D. R., \& Sarkissian, J. 2002, ApJ, 567, L71

Chevalier, R. A. 1982, ApJ, 259, 302

Chevalier, R. A. 2005, ApJ, 619, 839

Couderc, P. 1939, Ann. d'Astrophys., 2, 271

Douvion, T., Lagage, P. O., Cesarsky, C. J., Dwek, E. 2001, A\&A, 373, 281

Draine, B. T., \& Lee, H. M. 1984, ApJ, 285, 89

Draine, B. T., 2003, ApJ, 598, 1017

Dwek, E., Petre, R., Szymkowiak, A., \& Rice, W. L. 1987, ApJ, 320, 27

Dwek, E., Foster, S. M., \& Vancura, O. 1996, ApJ, 457, 244 
Dwek, E. \& Arendt, R. G. 2008, ApJ, 685, 976

Edo, O. 1983, PhD Dissertation, Dept. of Physics, University of Arizona

Fesen, R. A. \& Gunderson, K. S. 1996, ApJ, 470, 967

Gaensler, B. M. \& Wallace, B. J. 2003, ApJ, 594, 326

Gaustad, J. E., McCullough, P. R., Rosing, W., \& Van Buren, D. 2001, PASP, 113, 1326

Ghavamian, P., Hughes, J, P. \& Williams, T. B. 2005, ApJ, 635, 365

Ghavamian, P., Raymond, J. C., Blair, W. P., Long, K. S., Tappe, A., Park, S., Winkler, P. F. 2009, ApJ, in press

Glassgold, A. E., Najita, J. R., \& Igea, J. 2007, ApJ, 656, 515

Gonzalez, M., \& Safi-Harb, S. 2003, ApJ, 583, 91

Goss, W. M., Shaver, P. A., Zealey, W. J., Murdin, P., \& Clark, D. H. 1979, MNRAS, 188, 357

Green, D. A., Tuffs, R. J., \& Popescu, C. C. 2004, MNRAS, 355, 1315

Hines, D. C., et al. 2004, ApJS, 154, 290

Hughes, J. P., et al. 2003, ApJ, 591, L139

Hui, C. Y., \& Becker, W. 2006, A\&A, L457, 33

Hwang, U., et al. 2004, ApJ, 615, 117

Jones, A. P. 2004 in ASP Conf. Ser. 309, Astrophysics of Dust, ed. A. N. Witt, G. C. Clayton, \& B. T. Draine (San Francisco: ASP), 347

Kawada, M., et al. 2007, PASJ, 59, 389

Koo, B.-C., Moon, D.-S., Lee, H.-G., Lee, J.-J., \& Matthews, K. 2007, ApJ, 657, 308

Krause, O., et al. 2005, Science, 308, 1604

Laor, A., \& Draine, B. T. 1993, ApJ, 402, 441

Lockhart, I. A., Gross, W. M., Caswell, J. L., \& McAdam, W. B. 1977, MNRAS, 179, 147

Mathis, J. S., Rumpl, W., \& Nordsieck, K. H. 1977, ApJ, 217, 425 
Matzner, C. D., \& McKee, C. F. 1999, ApJ, 510, 379

Moon, D.-S., et al. 2004, ApJ, 610, L33

Moon, D.-S., et al. 2009, ApJ, 703, L81

Murakami, H., et al. 2007, PASJ, 59, 369

Murdin, P. \& Clark, D. H. 1979, MNRAS, 189, 501

Ng, G.-Y. \& Romani, R. W. 2007, ApJ, 660, 1357

Nozawa, T., Kozasa, T., Umeda, H., Maeda, K. \& Nomoto, K. 2003, ApJ, 598, 785

Nozawa, T., Kozasa, T., \& Habe, A. 2006, ApJ, 648, 435

Ohyama, Y., et al. 2007, PASJ 59, 411

Onaka, T., et al. 2007, PASJ 59, 401

Park, S., Roming, P. W. A., Hughes, J. P., Slane, P. O., Burrows, D. N., Garmire, G. P., \& Nousek, J. A. 2002, ApJ, 564, L39

Park, S., et al. 2004, ApJ, 602, L33

Park, S., Hughes, J. P., Slane, P. O., Burrows, D. N., Gaensler, B. M., \& Ghavamian, P. 2007, ApJ, 670, L121

Pittard, J. M., Dyson, J. E., Falle, S. A. E. G., \& Hartquist, T. W. 2001, A\&A, 375, 827

Rho, J., Kozasa, T., Reach, W. T., Smith, J. D., Rudnick, L., DeLaney, T., Ennis, J. A., Gomez, H., \& Tappe, A. 2008, ApJ, 673, 271

Rho, J., Reach, W. T., Tappe, A., Hwang, U., Slavin, J. D., Kozasa, T., \& Dunne, L., 2009, ApJ, 700, 579

Rodgers, A. W., Campbell, C. T., \& Whiteoak, J. B. 1960, MNRAS, 121, 103

Sakon, I., et al. 2008, Proc. of SPIE, 7010, 88

Semenov, D., Henning, Th., Helling, Ch., Ilgner, M., \& Sedlmayr, E. 2003, A\&A, 410, 611

Smith, J. D. T., Rudnick, L., Delaney, T., Rho, J., Gomez, H., Kozasa, T., Reach, W., \& Isensee, K. 2009, ApJ, 693, 713

Temim, T., et al. 2006, AJ, 132, 1610 
Tielens, A. G. G. M., McKee, C. F., Seab, C. G., \& Hollenbach, D. J. 1994, ApJ, 431, 321

Vancura, O., Raymond, J. C., Dwek, E., Blair, W. P., Long, K. S., \& Foster, S. 1994, ApJ, 431, 188

Wheeler, J. C., Maund, J. R., \& Couch, S. M. 2008, ApJ, 677, 1091

Williams, B. J., et al. 2006, ApJ, 652, L33

Williams, B. J., et al. 2008, ApJ, 687, 1054

Winkler, P. F. \& Long, K. S. 2006, AJ, 132, 360

Winkler, P. F., \& Petre, R. 2007, ApJ, 670, 635

Winkler, P. F., Twelker, K., Reith, C. N., \& Long, K. S. 2009, ApJ, 692, 1489

Zeiger, B. R., Brisken, W. F., Chatterjee, S., \& Goss, W. M. 2008, ApJ, 674, 271

Zubko, V., Dwek, E., \& Arendt, R. G. 2004, ApJS, 152, 211 


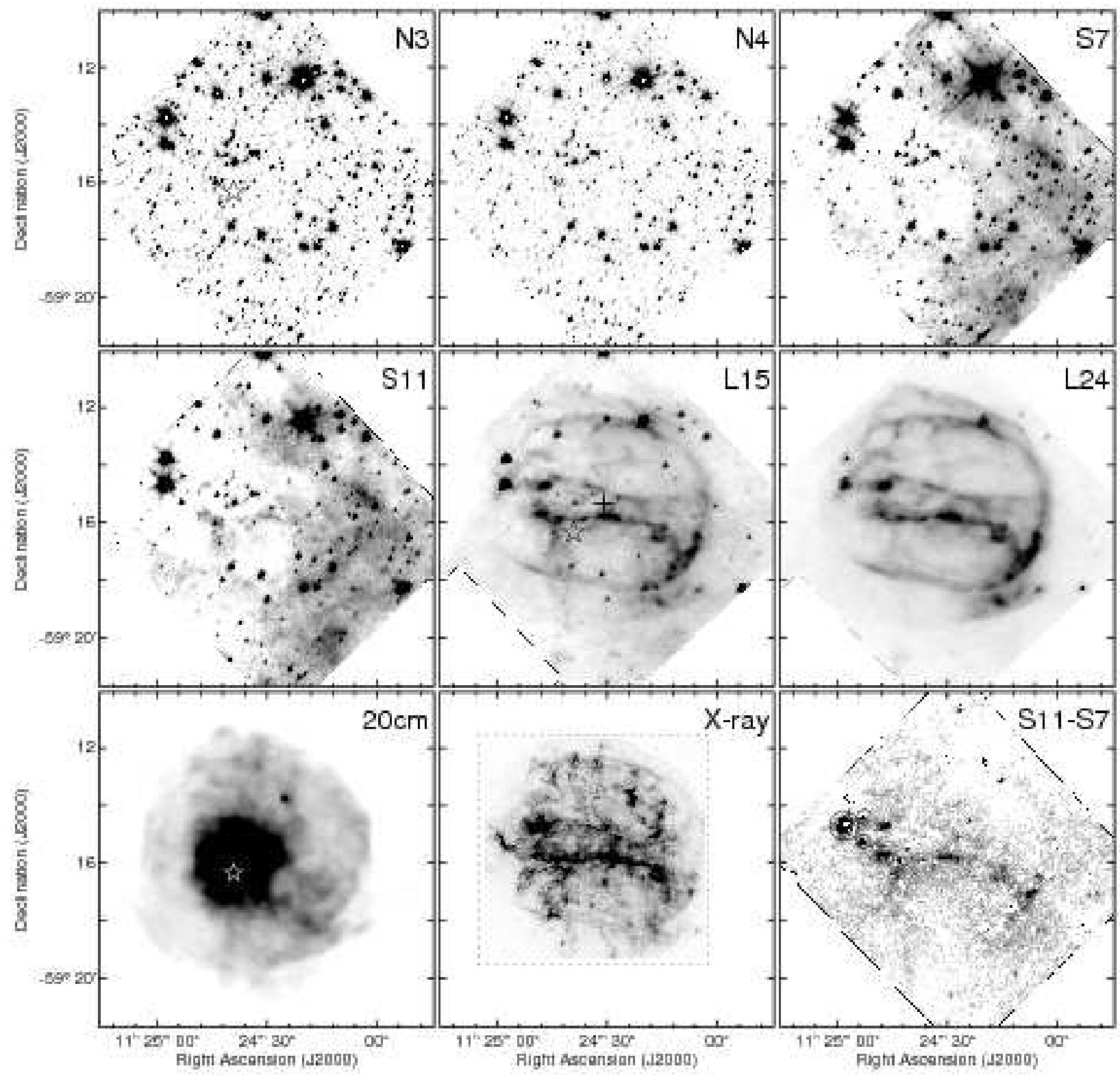

Fig. 1.- AKARI IRC images of G292.0+1.8 with the ATCA $20 \mathrm{~cm}$ continuum and Chandra X-ray map for comparison (Gaensler \& Wallace 2003; Park et al. 2002). The backgroundsubtracted S11-band image (S11-S7 difference image) is shown at the lower-right panel. The three circles close to the eastern end of the ER in the S11-S7 difference image indicate stellar sources. We mark the position of pulsar PSR J1124-5916 in N3-, L15-band (Hughes et al. 2003 ), and $20 \mathrm{~cm}$ continuum images by the star (Gaensler \& Wallace 2003). We also mark the center of OES by the plus sign in L15 image. The dotted box in the X-ray map indicates the imaging area of Chandra. The gray-scales of N3, N4, S7, S11, L15, L24 and S11-S7 bands are $0.5-3.0,0.5-3.0,5.3-6.3,15.8-16.7,23-26,29-40$, and $0-0.4 \mathrm{MJy} \mathrm{sr}^{-1}$, respectively. 


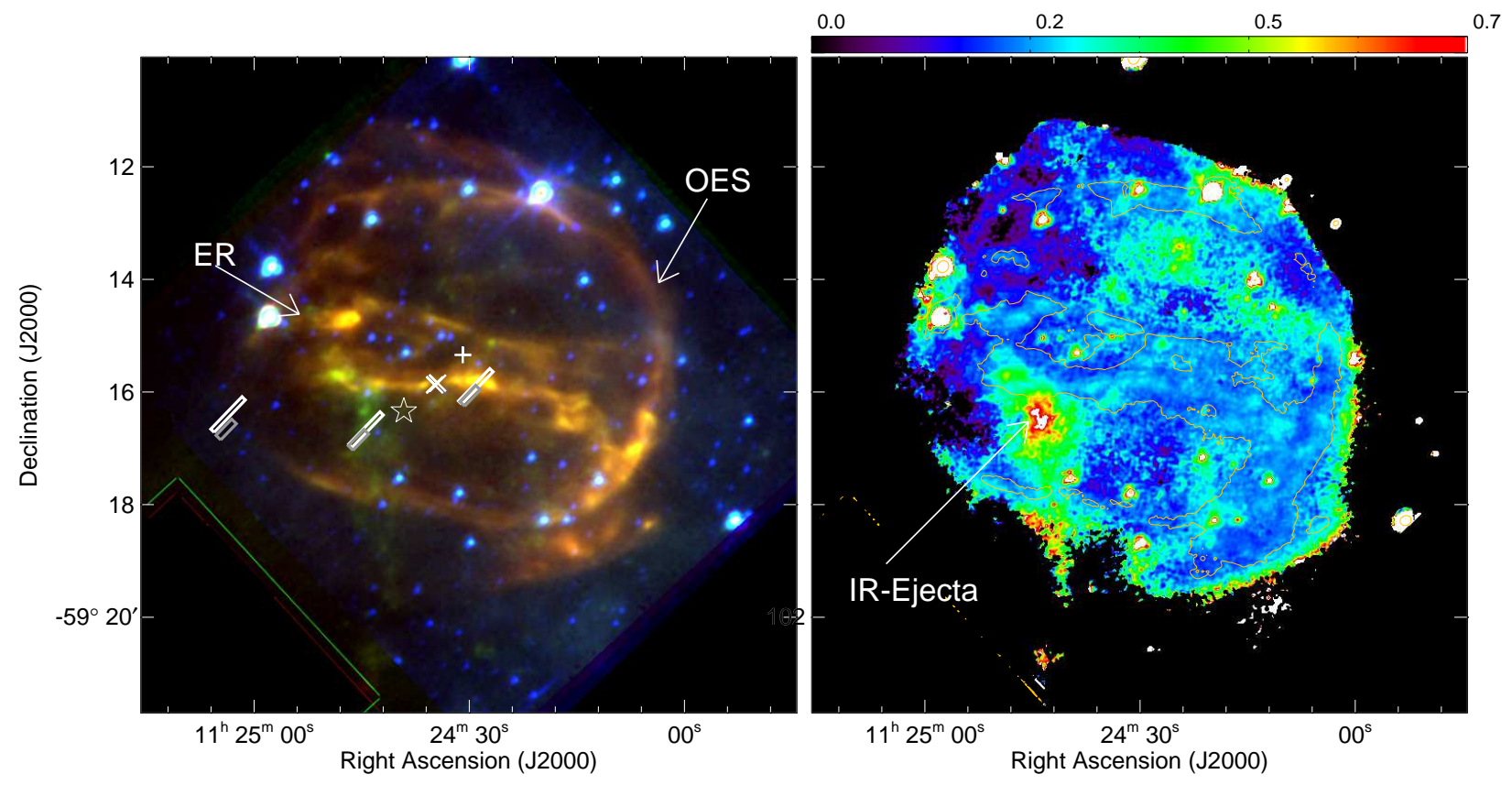

Fig. 2.- (Left) The three-color image of G292.0+1.8 produced from $24 \mu \mathrm{m}(\mathrm{R}), 15 \mu \mathrm{m}(\mathrm{G})$, and $7 \mu \mathrm{m}(\mathrm{B})$. The coordinates of equatorial, ejecta and background NG/SG slit positions are indicated by white box at $\left(11^{\mathrm{h}} 24^{\mathrm{m}} 29^{\mathrm{s}} .1,-59^{\circ} 15^{\prime} 54^{\prime \prime}\right),\left(11^{\mathrm{h}} 24^{\mathrm{m}} 44^{\mathrm{s}} .3,-59^{\circ} 16^{\prime} 40^{\prime \prime}\right)$, and $\left(11^{\mathrm{h}} 25^{\mathrm{m}} 03^{\mathrm{s}} .6,-59^{\circ} 16^{\prime} 24^{\prime \prime}\right)$, respectively. At the southwestern side of them, the three LG2 slit positions are indicated by gray box at $\left(11^{\mathrm{h}} 24^{\mathrm{m}} 29^{\mathrm{s}} .9,-59^{\circ} 16^{\prime} 04^{\prime \prime}\right),\left(11^{\mathrm{h}} 24^{\mathrm{m}} 45^{\mathrm{s}} .4,-59^{\circ} 16^{\prime} 51^{\prime \prime}\right)$, and $\left(11^{\mathrm{h}} 25^{\mathrm{m}} 03^{\mathrm{s}} .8,-59^{\circ} 16^{\prime} 40^{\prime \prime}\right)$, respectively. We mark the center of the OES by plus sign, both the dynamical center of optical ejecta knots (Winkler et al. 2009) and the center of outer radio plateau (Gaensler \& Wallace 2003) by crosses, and the position of pulsar PSR J11245916 by star (Hughes et al. 2003). The two cross symbols are almost coincident. (Right) The background subtracted 15 to $24 \mu \mathrm{m}$ ratio image. The pixels with low $24 \mu \mathrm{m}$ intensities are blanked out. The bright structures in $24 \mu \mathrm{m}$ images are shown by contour. The prominent features such as equatorial ring (ER), outer elliptical shell (OES), and IR-Ejecta are also labeled. 


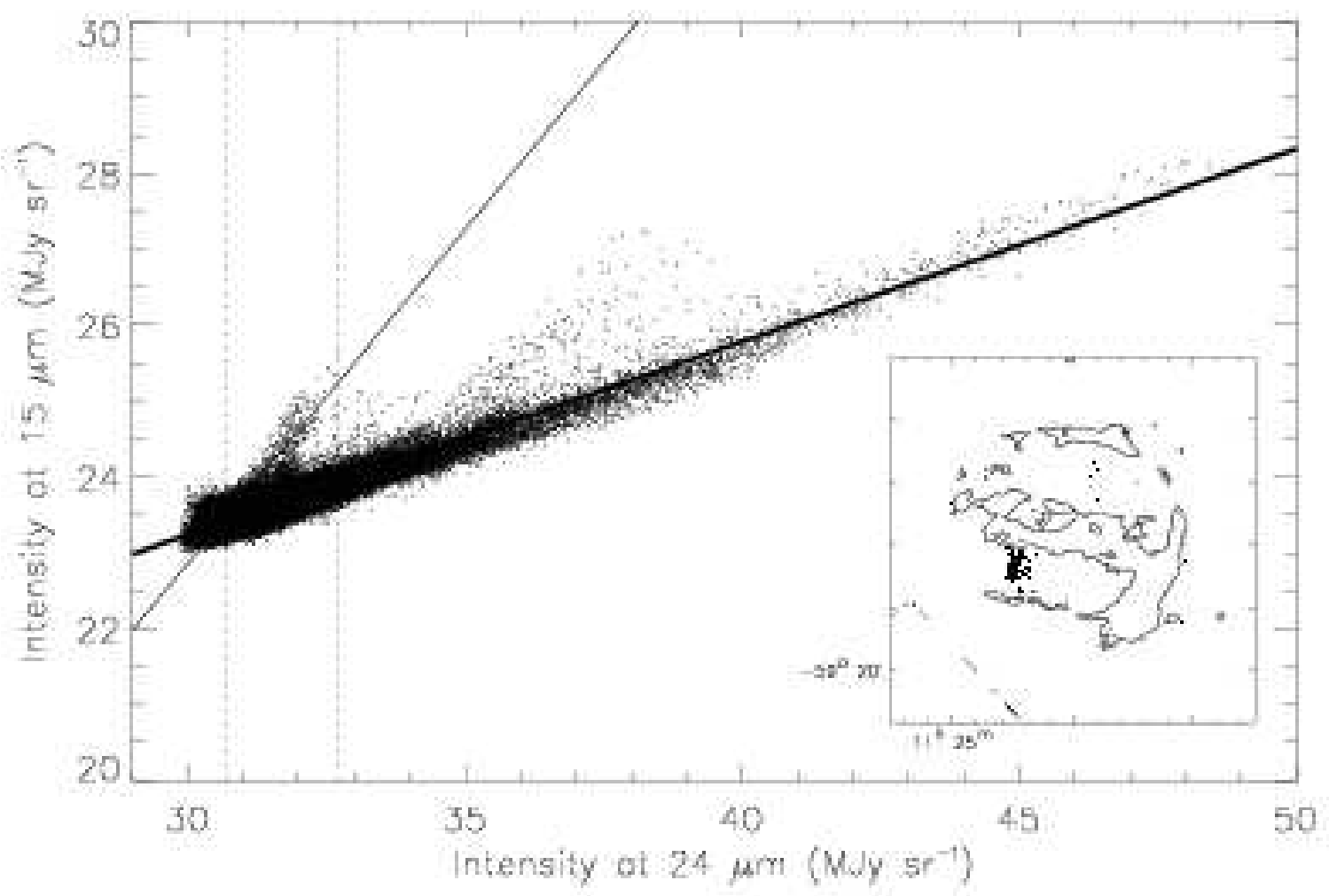

Fig. 3.- The L15 vs L24 band intensity plot. We mask the pixels with stellar emission using N3 band and the pixels with low intensity using L24 band. The vertical lines indicate the boundaries of the points with tight correlation (emission only from ejecta). The thick line is a linear fit using all pixels in the plot. The thin line is a linear fit using the high L15/L24 ratio region between two vertical lines. The position of high L15/L24 ratio pixels are marked in the inset in the lower-right corner superposed on the L24 contour. Black dots are the positions of the high L15/L24 ratio pixels between two vertical lines, and gray dots are those of high L15/L24 ratio pixels with L24 intensity $\gtrsim 33 \mathrm{MJy} \mathrm{sr}^{-1}$ (ejecta emission superposed on swept-up material). 


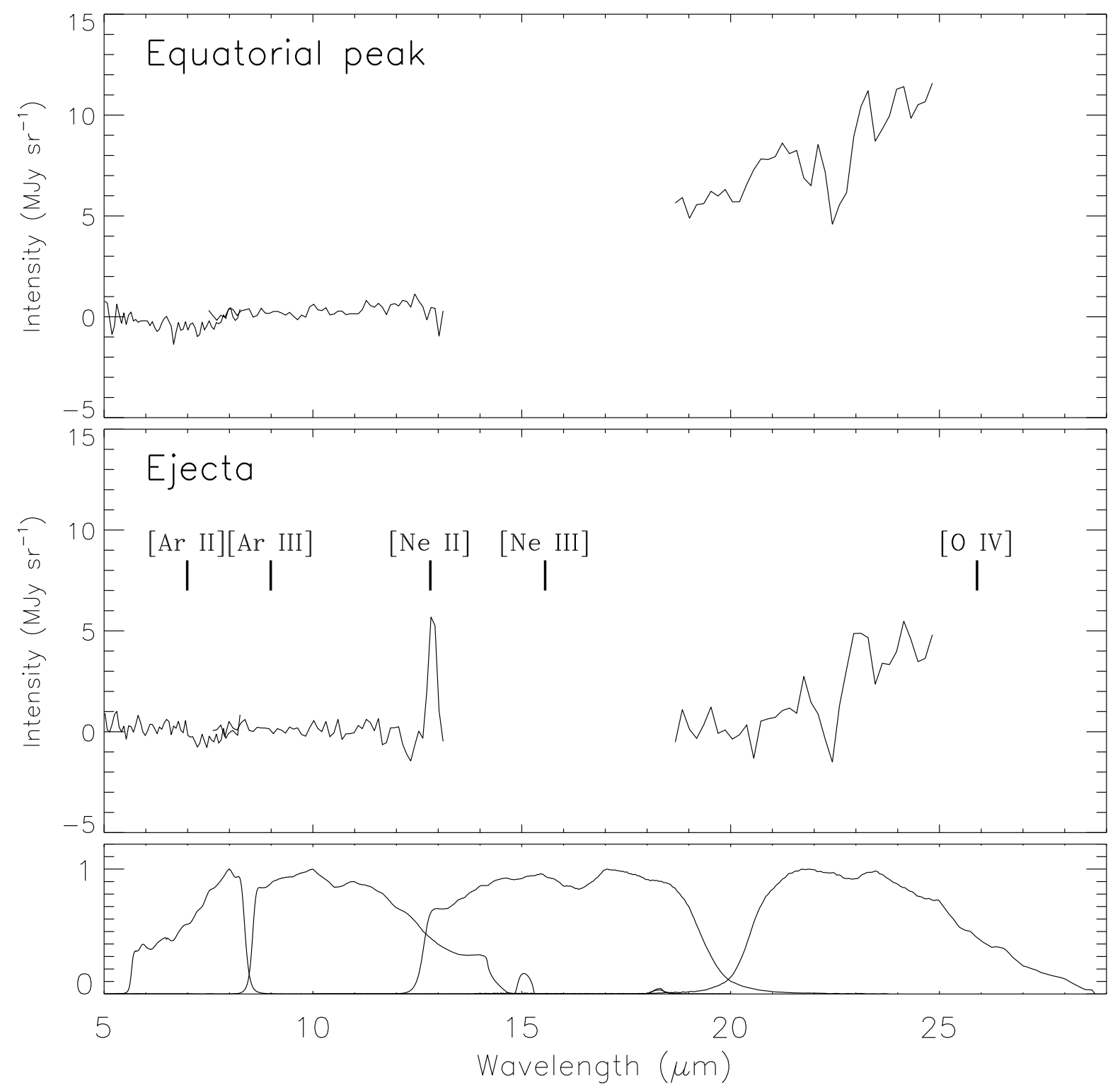

Fig. 4. - The background subtracted spectra obtained at the equatorial peak and ejecta positions. The MIR wavelength is covered by three elements: SG1 $(4.6-9.2 \mu \mathrm{m})$, SG2 (7.2$13.4 \mu \mathrm{m})$, and LG2 $(17.5-26.5 \mu \mathrm{m})$. Note that the positions of LG2 spectra is slightly shifted from those of SG spectra. Their positions and coordinates are indicated in Figure 2. The relative spectral responses of S7, S11, L15, and L24 imaging bands are indicated in bottom panel. 


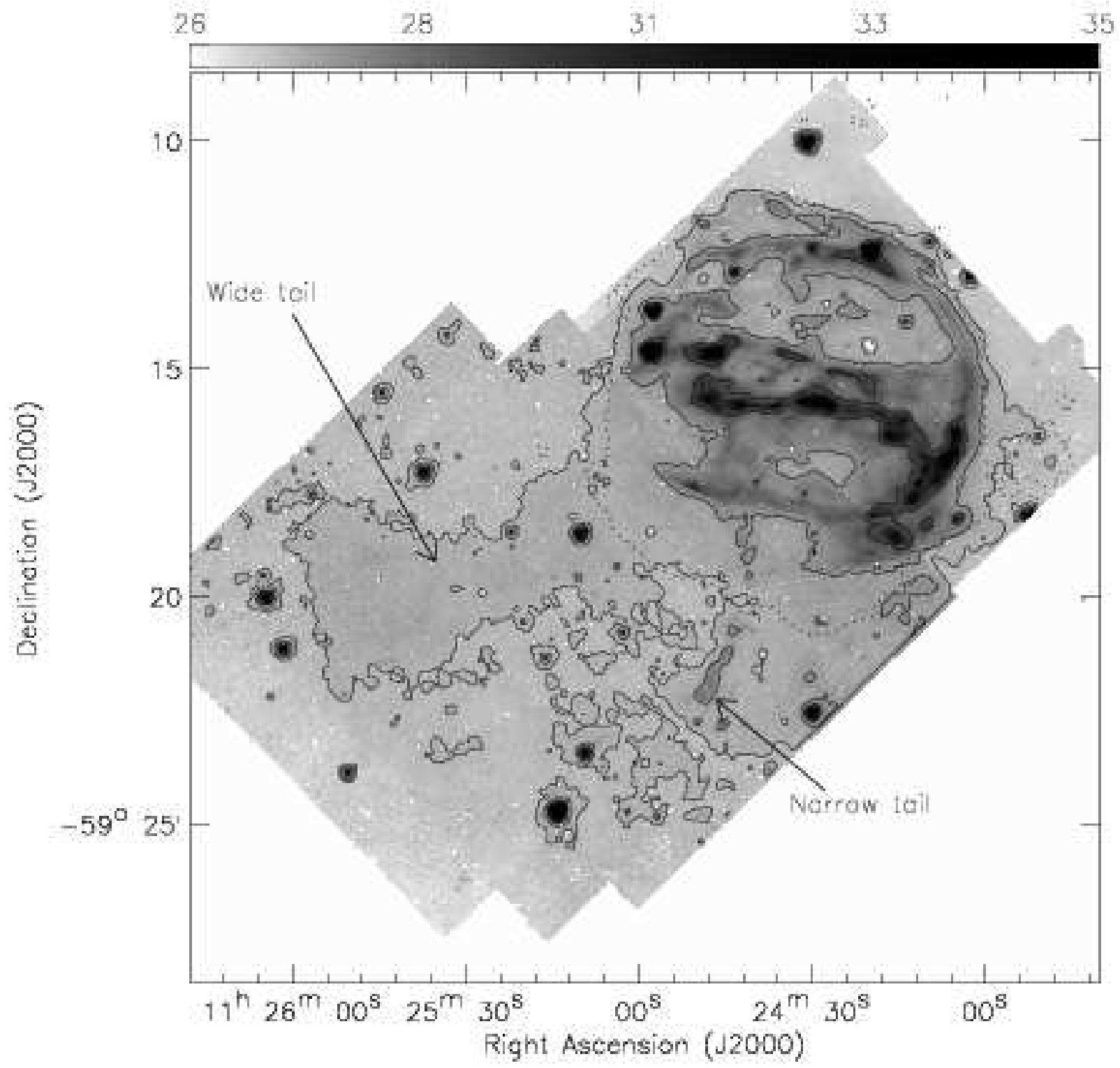

Fig. 5.- The large-scale L18W-band image of G292.0+1.8. Gray-scale range is indicated at the top. Contour levels are 27.0, 27.5, 29, and $31 \mathrm{MJy} \mathrm{sr}^{-1}$. The dotted contour is the ATCA $20 \mathrm{~cm}$ radio-continuum boundary (Gaensler \& Wallace 2003). The "Narrow tail" and "Wide tail" features outside shell boundary are labeled. 

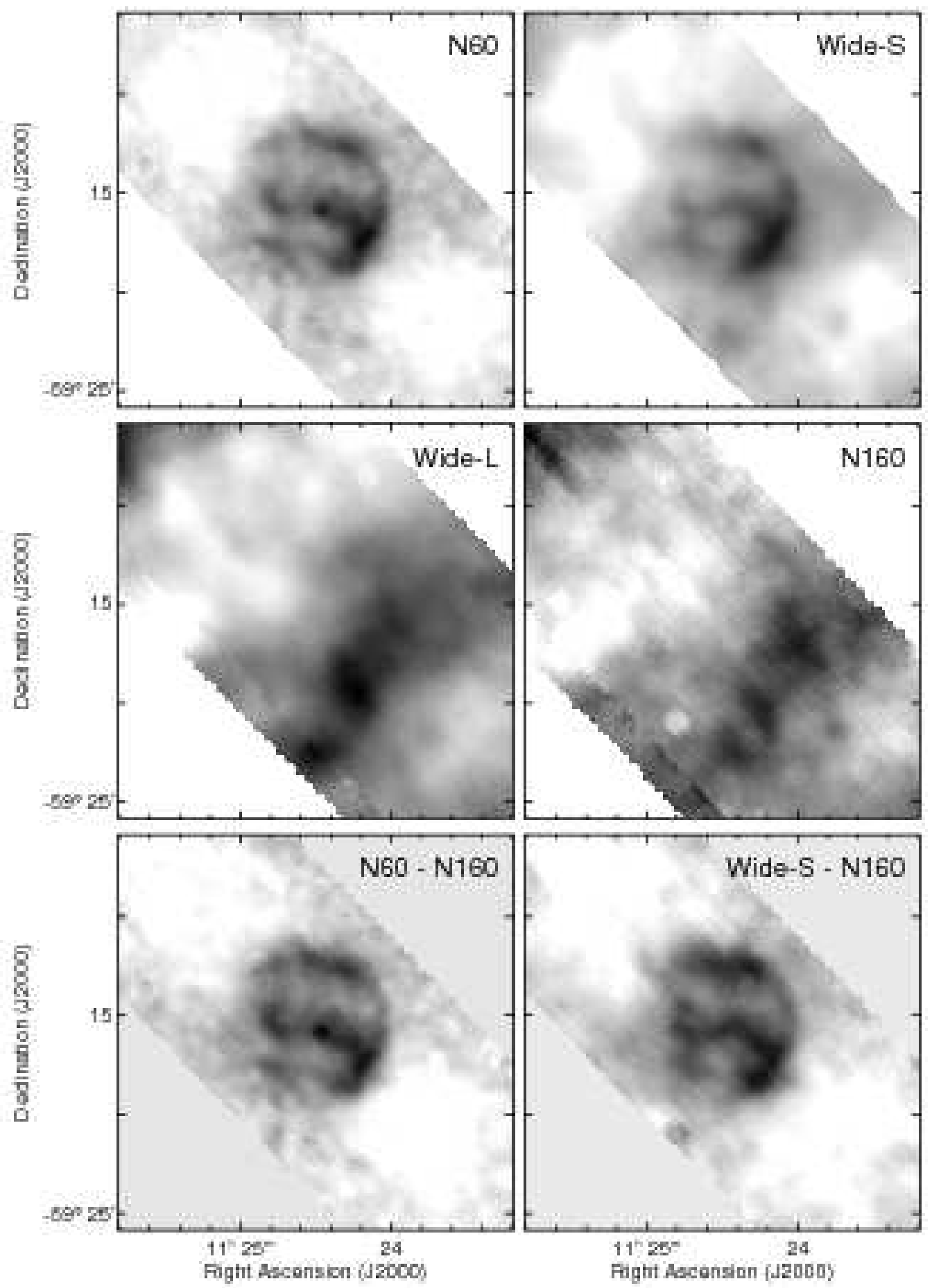

Fig. 6.- $-A K A R I$ FIS images of G292.0+1.8. The scan direction is northeast to southwest. The observed band is indicated at the upper-right corner in each panel. The background subtracted images are shown in the bottom panels. The gray-scales of N60, Wide-S, WideL, N160, N60-N160, and Wide-S-N160 bands are 18-28, 32-44, 70-88, 52-64, 0-9, and 0-8 $\mathrm{MJy} \mathrm{sr}^{-1}$, respectively. 


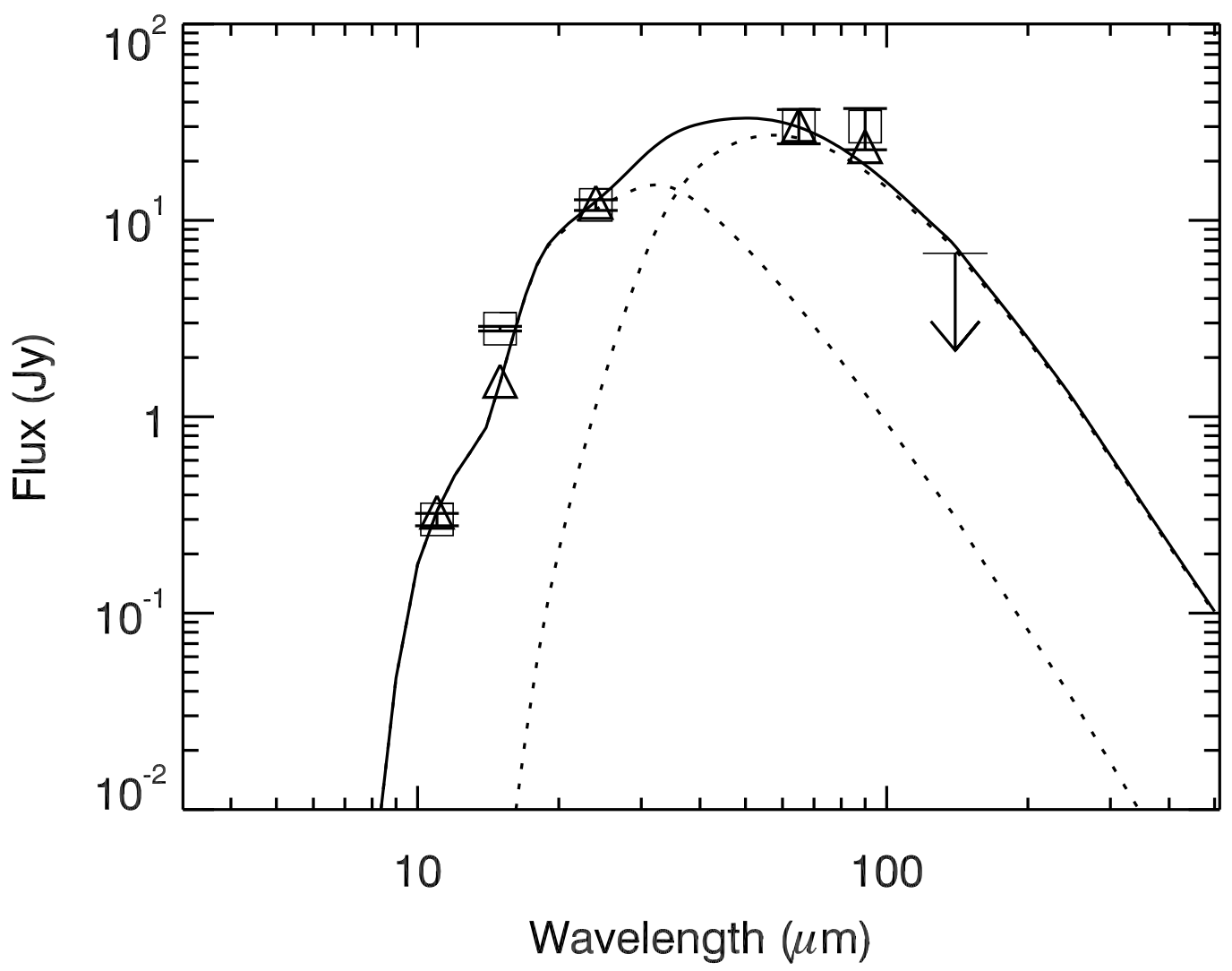

Fig. 7.- The measured total fluxes and the best fit of SED using the modified blackbody composed of two temperature dust components. The dust model is a mixture of carbonaceous and silicate interstellar grains of $R_{V}=3.1$. The boxes are the measured fluxes with errors and the triangles indicate the color-corrected fluxes. The downward arrow indicates the upper limit of $140 \mu \mathrm{m}$ flux. 

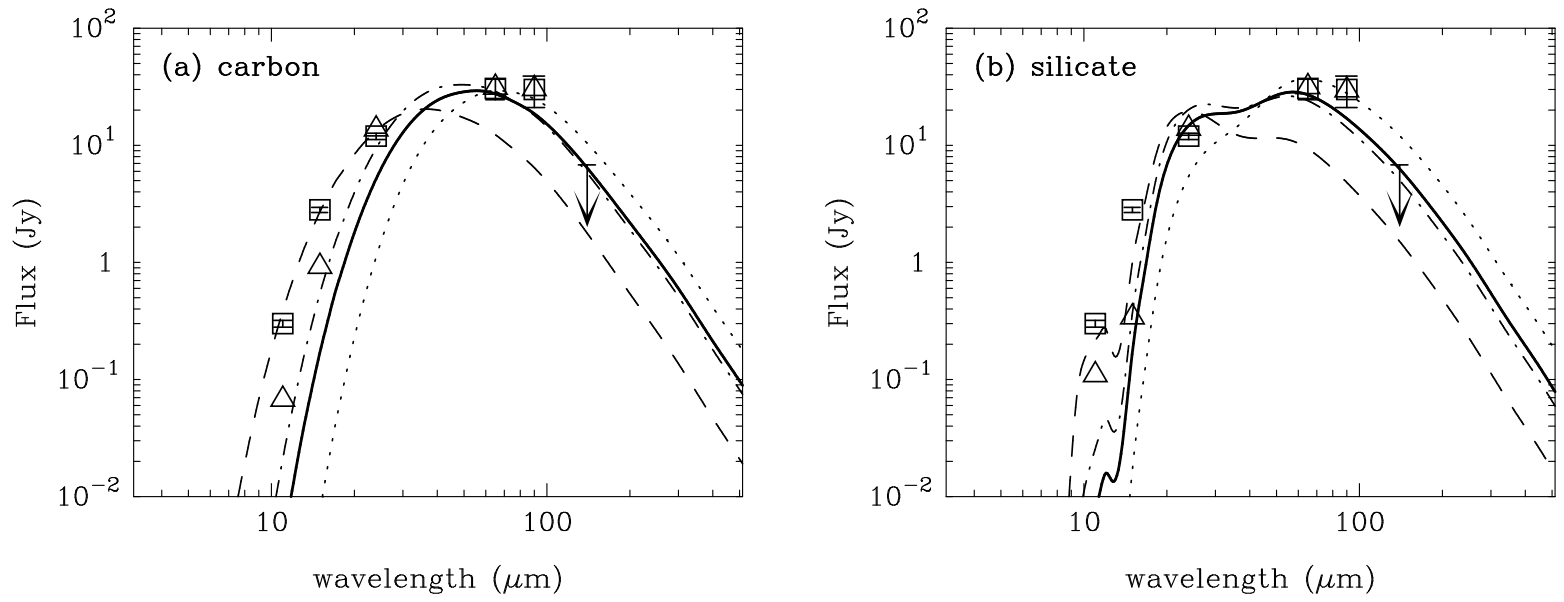

Fig. 8. - Comparison of the measured fluxes with the SED at 3,000 yrs obtained from the model calculations (See text for details.). The dust compositions are (a) amorphous carbon grains and (b) silicate grains. The dotted, solid, dashed-dotted and dashed lines indicate the results of simulations for $n_{\mathrm{H}, 0}=0.1,0.5,1$, and $10 \mathrm{~cm}^{-3}$. Symbols are the same as Figure 7 . 
Table 1. Summary of $A K A R I$ observations

\begin{tabular}{lccl}
\hline \hline \multicolumn{1}{c}{ Date } & \multicolumn{1}{c}{ ID $^{\mathrm{a}}$} & \multicolumn{1}{c}{ Mode } & \multicolumn{1}{c}{ Band } \\
\hline 2007. 1. 17. & 1400748 & IRC imaging & N3, N4, S7, S11 \\
2007. 1. 17. & 1400749 & IRC imaging & L15, L24 \\
2007. 1. 19. & 1400751 & FIS imaging & N60, Wide-S, Wide-L, N160 \\
2007. 7. 21. & 1402801 & IRC imaging & L18W \\
2007. 7. 20. & $1402839,41,43$ & IRC spectroscopy & NIR, MIR-S \\
2007. 7. 21.-22. & $1402840,42,44$ & IRC spectroscopy & MIR-L \\
\hline
\end{tabular}

a $A K A R I$ observational identification number. These observations are part of $A K A R I$ mission program, interstellar medium in our Galaxy and nearby galaxies (P.I.: H. Kaneda). 
Table 2. Characteristics of $A K A R I$ imaging observations

\begin{tabular}{|c|c|c|c|c|c|c|c|c|c|c|}
\hline \multirow{2}{*}{$\frac{\text { Instrument }}{\text { Channel }}$} & \multicolumn{6}{|c|}{ IRC } & \multicolumn{4}{|c|}{ FIS } \\
\hline & \multicolumn{2}{|c|}{$\begin{array}{c}\text { NIR } \\
512 \times 412 \mathrm{InSb}\end{array}$} & \multicolumn{2}{|c|}{$\begin{array}{c}\text { MIR-S } \\
256 \times 256 \mathrm{Si}: \mathrm{As}\end{array}$} & \multicolumn{2}{|c|}{$\begin{array}{c}\text { MIR-L } \\
256 \times 256 \mathrm{Si}: \mathrm{As}\end{array}$} & \multicolumn{2}{|c|}{$\begin{array}{c}\text { SW } \\
\text { Ge:Ga }\end{array}$} & \multicolumn{2}{|c|}{$\begin{array}{c}L W \\
\text { stressed Ge:Ga }\end{array}$} \\
\hline Band & N3 & N4 & S7 & $\mathrm{S} 11$ & $\mathrm{~L} 15$ & L24 & N60 & Wide-S & Wide-L & N160 \\
\hline Reference wavelength $(\mu \mathrm{m})$ & 3.2 & 4.1 & 7.0 & 11.0 & 15.0 & 24.0 & 65 & 90 & 140 & 160 \\
\hline Effective bandwidth $(\mu \mathrm{m})$ & 0.87 & 1.53 & 1.75 & 4.12 & 5.98 & 5.34 & 21.7 & 37.9 & 52.4 & 34.1 \\
\hline Pixel size $\left({ }^{\prime \prime}\right)$ & 1.46 & 1.46 & 2.34 & 2.34 & $2.51^{\mathrm{a}}$ & $2.51^{\mathrm{a}}$ & 26.8 & 26.8 & 44.2 & 44.2 \\
\hline FWHM $\left({ }^{\prime \prime}\right)$ & 4.0 & 4.2 & 5.1 & 4.8 & 5.7 & 6.8 & 37 & 39 & 58 & 61 \\
\hline
\end{tabular}

${ }^{\text {a }}$ Represents the width of pixel. The exact size is $2^{\prime \prime} .51 \times 2^{\prime \prime} .34$. 
Table 3. Characteristics of $A K A R I$ spectroscopic observations

\begin{tabular}{lcccc}
\hline \hline \multicolumn{1}{c}{ Channel } & NIR & \multicolumn{2}{c}{ MIR-S } & MIR-L \\
\hline Grism & NG & SG1 & SG2 & LG2 \\
Slit size $\left({ }^{\prime \prime} \times^{\prime}\right)$ & $5^{\prime \prime} \times 0 .^{\prime} 8$ & $5^{\prime \prime} \times 0 .^{\prime} 8$ & $5^{\prime \prime} \times 0 . .^{\prime} 8$ & $7^{\prime \prime} \times 0 . .^{\prime} 4$ \\
Wavelength coverage $(\mu \mathrm{m})$ & $2.5-5.0$ & $4.6-9.2$ & $7.2-13.4$ & $17.5-26.5$ \\
Resolving power & 120 & 53 & 50 & 48 \\
Exposure time $(\mathrm{s})$ & 400 & 196 & 245 & 442 \\
\hline
\end{tabular}


Table 4. Slit positions of $A K A R I$ spectroscopic observations

\begin{tabular}{lcc}
\hline \hline \multicolumn{1}{c}{ Slit position } & NG/SG1/SG2 & LG2 \\
\hline Equatorial peak & $\left(11^{\mathrm{h}} 24^{\mathrm{m}} 29^{\mathrm{s}} .1,-59^{\circ} 15^{\prime} 54^{\prime \prime}\right)$ & $\left(11^{\mathrm{h}} 24^{\mathrm{m}} 29^{\mathrm{s}} .9,-59^{\circ} 16^{\prime} 04^{\prime \prime}\right)$ \\
Ejecta peak & $\left(11^{\mathrm{h}} 24^{\mathrm{m}} 44^{\mathrm{s}} .3,-59^{\circ} 16^{\prime} 40^{\prime \prime}\right)$ & $\left(11^{\mathrm{h}} 24^{\mathrm{m}} 45^{\mathrm{s}} \cdot 4,-59^{\circ} 16^{\prime} 51^{\prime \prime}\right)$ \\
Background & $\left(11^{\mathrm{h}} 25^{\mathrm{m}} 03^{\mathrm{s}} \cdot 6,-59^{\circ} 16^{\prime} 24^{\prime \prime}\right)$ & $\left(11^{\mathrm{h}} 25^{\mathrm{m}} 03^{\mathrm{s}} .8,-59^{\circ} 16^{\prime} 40^{\prime \prime}\right)$ \\
\hline
\end{tabular}

Note. - The slit position angles were fixed to be $44^{\circ}$ by the satellite orbit. 
Table 5. IR measurements of G292.0+1.8

\begin{tabular}{lccccc}
\hline \hline \multirow{2}{*}{ Band } & \multicolumn{3}{c}{ Whole remnant } & \multicolumn{2}{c}{ IR-Ejecta } \\
\cline { 2 - 6 } & $\begin{array}{c}\text { Total Flux } \\
(\mathrm{Jy})\end{array}$ & $\begin{array}{c}\text { ER peak } \\
\left(\mathrm{MJy} \mathrm{sr}^{-1}\right)\end{array}$ & $\begin{array}{c}\text { OES peak } \\
\left(\mathrm{MJy} \mathrm{sr}^{-1}\right)\end{array}$ & $\begin{array}{c}\text { Ejecta flux } \\
\left(\mathrm{Jy}^{\mathrm{a}}\right)\end{array}$ & $\begin{array}{c}\text { Peak }^{\mathrm{b}} \\
\left(\mathrm{MJy} \mathrm{sr}^{-1}\right)\end{array}$ \\
\hline Measured flux/intensity & & & & & \\
S11 & $0.30(0.02)$ & $0.53(0.26)$ & $0.53(0.24)$ & $0.013(0.001)$ & $0.37(0.24)$ \\
L15 & $2.81(0.14)$ & $5.10(0.36)$ & $5.04(0.36)$ & $0.066(0.009)$ & $1.96(0.26)$ \\
L24 & $11.98(0.72)$ & $19.09(1.37)$ & $18.94(1.26)$ & $0.072(0.015)$ & $2.60(0.36)$ \\
N60 & $30.6(6.1)$ & $8.81(2.53)$ & $7.65(1.83)$ & $0.173(0.035)$ & $5.32(1.47)$ \\
Wide-S & $30.0(9.0)$ & $6.76(2.14)$ & $6.97(2.20)$ & $0.250(0.075)$ & $6.05(1.95)$ \\
Wide-L & $<6.8$ & $<6.50$ & $<6.58$ & $<0.048$ & $<6.87$ \\
Color & & & & & $0.19(0.13)$ \\
S11/L15 & $0.11(0.01)$ & $0.10(0.05)$ & $0.11(0.05)$ & $0.20(0.03)$ & $0.19(0.14)$ \\
L15/L24 & $0.23(0.02)$ & $0.27(0.03)$ & $0.27(0.03)$ & $0.92(0.23)$ & $0.75(0.14)$ \\
N60/Wide-S & $1.02(0.37)$ & $1.30(0.56)$ & $1.10(0.43)$ & $0.69(0.25)$ & $0.88(0.37)$ \\
Wide-S/Wide-L & $>4.42$ & $>1.04$ & $>1.06$ & $>5.25$ & $>0.88$ \\
\hline
\end{tabular}

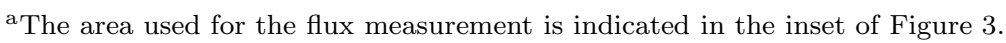

${ }^{b}$ Measured intensity at the peak of SG slit position. 
Table 6. Results of $A K A R I$ IRC spectroscopy

\begin{tabular}{cccc}
\hline \hline Line & $\begin{array}{c}\text { Wavelength } \\
(\mu \mathrm{m})\end{array}$ & $\begin{array}{c}\text { ER peak } \\
\left(\mathrm{erg} \mathrm{cm}^{-2} \mathrm{~s}^{-1} \mathrm{sr}^{-1}\right)\end{array}$ & $\begin{array}{c}\text { IR-Ejecta peak } \\
\left(\mathrm{erg} \mathrm{cm}^{-2} \mathrm{~s}^{-1} \mathrm{sr}^{-1}\right)\end{array}$ \\
\hline$[\mathrm{Ar} \mathrm{II}]$ & 7.0 & $<1.6 \times 10^{-6}$ & $<2.9 \times 10^{-6}$ \\
{$[\mathrm{Ar} \mathrm{III}]$} & 9.0 & $<4.0 \times 10^{-7}$ & $<1.1 \times 10^{-6}$ \\
{$[\mathrm{Ne} \mathrm{II}]$} & 12.8 & $<2.7 \times 10^{-6}$ & $2.8 \times 10^{-5}$ \\
\hline
\end{tabular}

Note. — Undetected lines below $3 \sigma$ detection limit. 